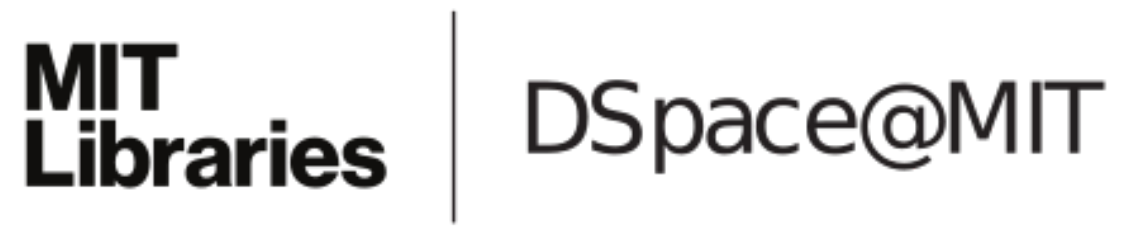

MIT Open Access Articles

Two-Dimensional Spectroscopy at Terahertz Frequencies

The MIT Faculty has made this article openly available. Please share how this access benefits you. Your story matters.

Citation: Topics in Current Chemistry. 2018 Jan 23;376(1):6

As Published: https://doi.org/10.1007/s41061-018-0185-4

Publisher: Springer International Publishing

Persistent URL: https://hdl.handle.net/1721.1/131483

Version: Author's final manuscript: final author's manuscript post peer review, without publisher's formatting or copy editing

Terms of use: Creative Commons Attribution-Noncommercial-Share Alike 


\title{
Two-dimensional spectroscopy at terahertz frequencies
}

\author{
Jian Lu, Xian Li, Yaqing Zhang, Harold Y. Hwang, Benjamin K. Ofori-Okai, and \\ Keith A. Nelson* \\ Department of Chemistry, Massachusetts Institute of Technology, Cambridge, \\ Massachusetts 02139, USA \\ kanelson@mit.edu
}

\begin{abstract}
Multidimensional spectroscopy in the visible and infrared spectral ranges has become a powerful technique to retrieve dynamic correlations and couplings in wideranging systems by utilizing multiple correlated light-matter interactions. Its extension to the terahertz $(\mathrm{THz})$ regime of the electromagnetic spectrum, where rich material degrees of freedom reside, however, has been progressing slowly. This chapter reviews some of the THz-frequency two-dimensional (2D) spectroscopy techniques and experimental results realized in recent years. Examples include gas molecule rotations, spin precessions in magnetic systems, and liquid molecular dynamics studied by $2 \mathrm{D} \mathrm{THz}$ or hybrid 2D THz-Raman spectroscopy techniques. The methodology shows promising applications to different THz-frequency degrees of freedom in various chemical systems and processes.
\end{abstract}

Keywords Terahertz; gas-phase molecular rotations; liquid-phase molecular dynamics; magnetic resonances

\section{Contents}

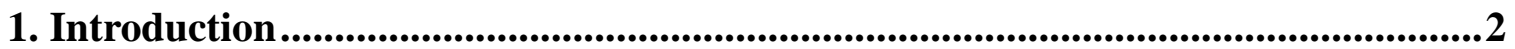

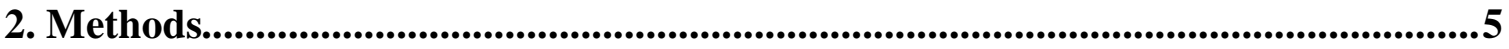

2.1 THz pulse generation and signal detection .........................................................5

2.1.1 Intense $\mathrm{THz}$ pulse generation methods ...............................................

2.1.2 THz time-domain detection by electro-optic sampling ............................. 8

2.1.3 Optical detection methods .......................................................... 9

2.2 Nonlinear 2D THz spectroscopy methods ..........................................................9

2.2.1 Pulse sequences in 2D THz and 2D THz-Raman spectroscopies ...................9

2.2.2 Collinear phase matching and differential chopping detection ..................... 12 
2.3 Experimental setups for 2D THz and 2D THz-Raman spectroscopies .13

3. 2D THz rotational spectroscopy of gas-phase molecules........................................16

3.1 Molecular orientation induced by THz pulses .......................................16

3.2 Molecular alignment induced by THz pulses and two-quantum THz-THzoptical rotational spectroscopy ........................................................................18

3.3 2D THz rotational spectroscopy of acetonitrile ........................................22

3.4 Extensions of 2D THz rotational spectroscopy ...............................................227

4. 2D THz and hybrid 2D THz-Raman vibrational spectroscopies .........................27

4.1 2D THz spectroscopy of phonons in semiconductors...................................28

4.2 2D THz-THz-Raman spectroscopy of intramolecular vibrations in liquids ....32

4.3 2D Raman-THz spectroscopy of water and aqueous salt solutions..................35

4.4 Extensions of 2D THz and 2D THz-Raman vibrational spectroscopies...........38

5. 2D THz magnetic resonance spectroscopy ........................................................38

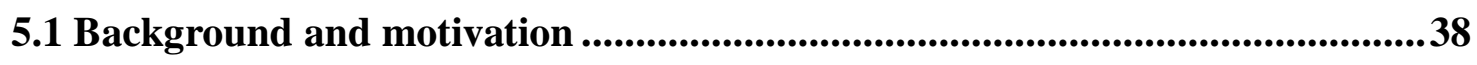

5.2 2D THz spectroscopy of collective spin waves............................................39

5.3 Extensions of 2D THz EPR spectroscopy ...............................................44

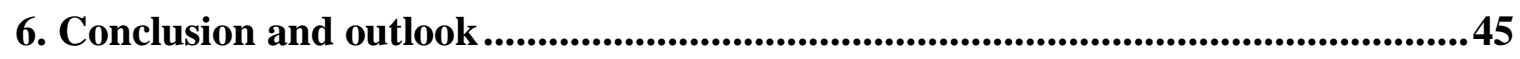

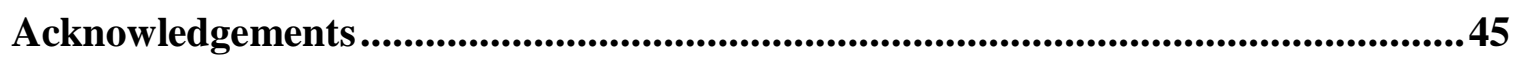

References............................................................................................................46

\section{Introduction}

Recent years have witnessed increasing interest in two-dimensional spectroscopy in many regions of the spectrum. Two-dimensional infrared (2D IR) vibrational spectroscopy [1-4] is a powerful technique for studying structural dynamics and correlations between coupled molecular motions in chemical and biological systems such as water [5], proteins [6], and DNA [7]. With visible light, multidimensional electronic spectroscopies [8] have been applied to probe the high-order correlations of excitons in quantum wells [9, 10] and light-harvesting complexes [11-13]. Recently, 2D electronic spectroscopy has been extended across the visible spectrum and into the ultraviolet regime $[8,14]$. In the most common $2 \mathrm{D}$ spectroscopy experiments, three resonant 
interactions between incident light fields and the sample generate a nonlinear signal field that is fully characterized through heterodyne mixing with a reference or local-oscillator field and either time- or frequency-resolved measurement of the superposition. By varying the relative time delays and relative phases between incident fields and measuring the effects on the signal field, dynamical information about sample coherences and populations, couplings between different modes, and a great deal more can be learned. Variation of the time interval between two phase-coherent incident fields provides a second dimension along which the nonlinear signal can be measured, usually displayed along a second frequency axis after Fourier transformation of the signal as a function of the relative (inter-pulse) delay. The results often reveal features that remain hidden in conventional 1D linear spectra.

The terahertz $(\mathrm{THz})$ regime of the electromagnetic spectrum is overlapped with a rich variety of material degrees of freedom including rotations of polar gas molecules, lattice vibrations in solids, molecular dynamics of liquids, spin dynamics in materials with magnetic order and/or high magnetic anisotropy, and many others [15]. Linear THz timedomain spectroscopy allows access to both the amplitude and phase of the $\mathrm{THz}$ electric field after its interaction with the sample, from which the real and imaginary parts of the dielectric function can be retrieved simultaneously without referring to the KramersKronig relation [15]. Linear $\mathrm{THz}$ time-domain spectroscopy has found wide-ranging applications in the characterization of molecular and material systems relevant to chemistry, biology, and physics. In such experiments, $\mathrm{THz}$ pulses are typically generated by femtosecond laser pulses that either undergo optical rectification (OR) in nonlinear optical crystals such as zinc telluride (ZnTe), gallium phosphate (GaP), or gallium arsenide $(\mathrm{GaAs})$, or that generate ultrafast photocurrent in biased photoconductive antennas [15]. The achievable electric field strength is generally less than $10 \mathrm{kV} / \mathrm{cm} \mathrm{[16],}$ which in most samples precludes any significant nonlinear light-matter interactions. The absence of strong tabletop $\mathrm{THz}$ sources placed early $\mathrm{THz}$ spectroscopy exclusively in the linear response region [17-19].

With the advent of strong tabletop $\mathrm{THz}$ sources, the subfield of nonlinear $\mathrm{THz}$ spectroscopy has been growing rapidly in the past decade. Nowadays, intense singlecycle $\mathrm{THz}$ pulses spanning the low $\mathrm{THz}$ region between 0.1 and $3 \mathrm{THz}$ with pulse energies of several $\mu \mathrm{J}$ and electric field strengths as high as $1 \mathrm{MV} / \mathrm{cm}$ (corresponding to magnetic field strengths of $0.33 \mathrm{~T}$ ) have been routinely generated by OR of Ti:Sapphire laser pulses in lithium niobate $\left(\mathrm{LiNbO}_{3}\right)$ crystals $[20,21]$. THz pulses of $10 \mathrm{MV} / \mathrm{cm}$ electric fields (corresponding to multi-tesla magnetic fields), higher center frequencies and broader bandwidths have been recently demonstrated by OR of near-IR fs pulses with center wavelengths between 1.2 and $1.5 \mu \mathrm{m}$ in novel organic nonlinear optical crystals [22-24]. The rapid development of intense $\mathrm{THz}$ sources has enabled coherent THz spectroscopy and control over electronic, orbital, lattice and spin degrees of freedom in various systems $[25,26]$. 
Compared to the well-established research areas of multidimensional spectroscopy in the visible and IR frequency ranges, the methodology and application of $2 \mathrm{D} \mathrm{THz}$ spectroscopy are still in a nascent stage. In recent years, there have been developments of 2D THz techniques which led to the first demonstrations of $2 \mathrm{D}$ multi- $\mathrm{THz}$ and $2 \mathrm{D} \mathrm{THz}$ spectroscopies in the study of mostly nonresonant electronic and some lattice vibrational responses with strong nonlinearities in condensed-matter systems. Examples include carrier dynamics in graphene [27], correlations between carriers and phonons in quantum wells [27, 28], THz nonlinear frequency mixing in $\mathrm{LiNbO}_{3}$ [29], and coherent cyclotron resonance nonlinear mixing in a $2 \mathrm{D}$ electron gas [30]. Demonstrations of $2 \mathrm{D} \mathrm{THz}$ spectroscopy more directly relevant to chemistry include $2 \mathrm{D} \mathrm{THz}$ rotational spectroscopy of molecules in the gas phase [31, 32], 2D THz vibrational spectroscopy of phonons in a semiconductor [33, 34], and 2D THz magnetic resonance spectroscopy of magnons [35, 36]. In the first of these, THz photon echo signals have been revealed for the first time in the gas phase and the full set of THz third-order $\left(\chi^{(3)}\right)$ nonlinear responses have been mapped into 2D spectra. In the second example, the first demonstration of $2 \mathrm{D} \mathrm{THz}$ spectroscopy using three separate pulses has been realized which reveals strong nonresonant nonlinearity beyond $\chi^{(3)}$ responses associated with the two-phonon quantum coherences in the system. The last example, though conducted on magnons in an antiferromagnetic crystal, is directly relevant for the development of $2 \mathrm{D} \mathrm{THz}$ electron paramagnetic resonance (EPR) spectroscopy and the study of chemical and biological systems which have spin resonances in the $\mathrm{THz}$ regime. $\mathrm{THz}$ pulses combined with optical excitation and detection through non-resonant Raman processes have enabled demonstrations of hybrid 2D THz-Raman spectroscopies. Hybrid 2D THz-Raman studies have revealed couplings among the intra-molecular vibrational modes in halogenated liquids [37, 38], and the inter-molecular dynamics of the hydrogen-bond network in water [39] and aqueous salt solutions [40] through THz photon echoes.

This chapter is organized as follows. In the Methods section, the basic techniques for intense $\mathrm{THz}$ pulse generation and time-domain signal detection are reviewed. The methods involving $2 \mathrm{D} \mathrm{THz}$ spectroscopy in collinear geometry and typical pulse sequences are discussed. As there are key differences between the techniques used in 2D $\mathrm{THz}$ and 2D IR or visible spectroscopies, we will elaborate the 2D THz methods in detail and frequently make comparisons with their analogs in the IR and visible. In what follows, we will review $2 \mathrm{D} \mathrm{THz}$ rotational spectroscopy, $2 \mathrm{D} \mathrm{THz}$ vibrational spectroscopy, 2D THz-Raman spectroscopies and 2D THz magnetic resonance spectroscopy in detail. Lastly, a summary and a brief outlook of the directions to which 2D THz spectroscopy can lead are presented. 


\section{Methods}

\subsection{THz pulse generation and signal detection}

As 2D spectroscopies involve nonlinear signal generation and detection, experiments require strong excitation sources and sensitive detection schemes. Intense $\mathrm{THz}$ pulse generation mainly relies on OR of strong fs laser pulses in a nonlinear optical crystal. The typical picosecond (ps) to sub-ps durations of $\mathrm{THz}$ pulses allow detection of their electric field profiles in the time domain, gated by fs laser pulses.

\subsubsection{Intense THz pulse generation methods}

OR is a second-order $\left(\chi^{(2)}\right)$ nonlinear process. Typically, a broadband fs laser pulse at $800 \mathrm{~nm}$ from a Ti:Sapphire amplifier is used to pump a nonlinear optical crystal in collinear geometry. Field components at nearby frequencies, $E\left(\omega_{1}\right)$ and $E\left(\omega_{2}\right)$, within the laser pulse bandwidth undergo difference-frequency mixing and generate electromagnetic radiation centered at $\left|\omega_{1}-\omega_{2}\right|$, which is in the THz frequency range. In the perturbative regime, the THz-frequency fields are radiated by a $\chi^{(2)}$ polarization $P^{(2)}$ [41] given by,

$$
P^{(2)}\left(\omega_{\mathrm{THz}}\right)=\chi^{(2)}\left(\omega_{\mathrm{THz}} ; \omega_{1}, \omega_{2}\right) E\left(\omega_{1}\right) E\left(\omega_{2}\right) .
$$

Similar to other nonlinear processes [41], the $\chi^{(2)}$ nonlinear coefficient of the crystal and the phase matching of the optical pump and the generated $\mathrm{THz}$ pulses in the crystal are crucial for efficient generation of the $\mathrm{THz}$ fields. The phase-matching condition for $\mathrm{THz}$ generation by $\mathrm{OR}$ with collinear optical and $\mathrm{THz}$ wavevectors is given by a scalar equation,

$$
k\left(\omega_{\mathrm{THz}}\right)=k\left(\omega_{1}\right)-k\left(\omega_{2}\right) \approx k\left(\omega_{2}\right)+d k\left(\omega_{2}\right) / d \omega \cdot \omega_{\mathrm{THz}}-k\left(\omega_{2}\right),
$$

where $k$ is the wavevector magnitude, $\omega$ the angular frequency, and $\omega_{\mathrm{THz}}=\left|\omega_{1}-\omega_{2}\right|$ is satisfied. From the perspective of photons, $\omega_{\mathrm{THz}}=\left|\omega_{1}-\omega_{2}\right|$ dictates photon energy conservation and Eq. (2) dictates photon quasi-momentum conservation. The index matching condition can be obtained from Eq. (2), and is given by

$$
n_{\mathrm{THz}}=n_{\mathrm{op}}^{\mathrm{g}}
$$

where $n_{\mathrm{THz}}=c \cdot k\left(\omega_{\mathrm{THz}}\right) / \omega_{\mathrm{THz}}$ is the $\mathrm{THz}$ refractive index and $n_{\mathrm{op}}^{\mathrm{g}}=c \cdot d k\left(\omega_{2}\right) / d \omega$ the optical group index in the nonlinear optical crystal ( $c$ is the speed of light in vacuum).

Inorganic nonlinear crystals including $\mathrm{ZnTe}, \mathrm{GaP}$, and $\mathrm{GaAs}$ have optimal indexmatching between $800 \mathrm{~nm}$ and THz pulses. Phase matching can be satisfied in a simple collinear geometry and the experimental implementation is hence straightforward. But these crystals have relatively small $\chi^{(2)}$ nonlinear coefficients and large-area crystals are 
typically required for strong $\mathrm{THz}$ pulse generation [16]. $\mathrm{LiNbO}_{3}$ has a large $\chi^{(2)}$ nonlinear coefficient, but the large phase mismatch between $800 \mathrm{~nm}$ and $\mathrm{THz}$ pulses in $\mathrm{LiNbO}_{3}$ leads to inefficient $\mathrm{THz}$ generation in collinear geometry. To circumvent the phasematching problem and utilize the large $\chi^{(2)}$ coefficient in $\mathrm{LiNbO}_{3}$, a non-collinear phasematching scheme involving tilting of the $800-\mathrm{nm}$ pulse intensity front to match the $\mathrm{THz}$ wavefront has been developed [20,21, 42, 43], which enabled the rapid proliferation of tabletop nonlinear THz spectroscopy experiments [25].

The geometry for the tilted-pulse-front method is shown schematically in Fig. 1(a). The broadband fs pump pulses from the Ti:Sapphire laser with a flat intensity front are incident onto a grating. The first-order diffraction which has a tilted intensity front is collected and imaged into a specially cut $\mathrm{LiNbO}_{3}$ crystal. The demagnification of the imaging lens adjusts the tilt angle of the intensity front for optimal phase matching. Once achieved, the wave front of the generated $\mathrm{THz}$ pulses is parallel to the intensity front of the pump pulses. They travel at the same velocity along the direction normal to the pump intensity front, and the $\mathrm{THz}$ field amplitude becomes coherently enhanced. As a result of this non-collinear phase matching, strong $\mathrm{THz}$ pulses with $\mu \mathrm{J}$ energies can be achieved at the output facet of the $\mathrm{LiNbO}_{3}$ crystal. Typical pump-to-THz energy conversion efficiency in $\mathrm{LiNbO}_{3}$ using the tilted-pulse-front method is on the order of $0.1 \%$ at room temperature. The generated $\mathrm{THz}$ pulses are collimated and focused to a submillimeter spot which can result in single-cycle $\mathrm{THz}$ electric fields of $1 \mathrm{MV} / \mathrm{cm}$ strength and bandwidth ranging from 0.1 to $3 \mathrm{THz}$ [21]. The focused $\mathrm{THz}$ fields are used to excite and interrogate the sample.
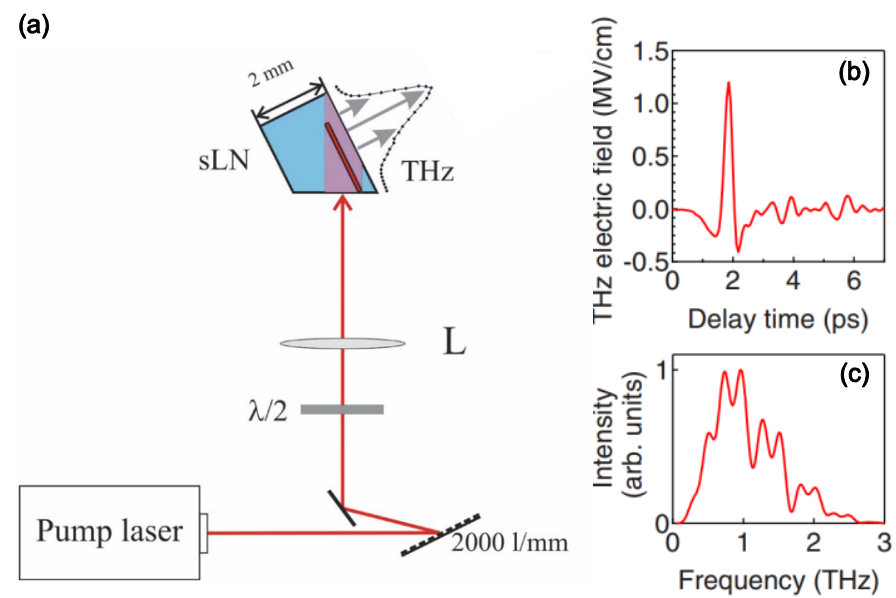

Fig. 1 (a) Schematic illustration of the setup for $\mathrm{THz}$ generation in a stoichiometric $\mathrm{LiNbO}_{3}$ (sLN) crystal using the tilted-pulse-front method. (b) and (c) Single-cycle THz electric field waveform (b) and spectrum (c) generated in $\mathrm{LiNbO}_{3}$. (from [21, 43]) 
Strong $\mathrm{THz}$ pulses can also be generated from organic nonlinear optical crystals with high $\chi^{(2)}$ nonlinear coefficients such as 2-(3-(4-hydroxystyryl)-5,5-dimethylcyclohex-2enylidene)malononitrile (OH1), and 4- $N, N$-dimethylamino- $4^{\prime}-N^{\prime}$-methyl-stilbazolium 2,4,6-trimethylbenzenesulfonate (DSTMS) [23]. Collinear phase matching between $\mathrm{THz}$ and pump pulses is achieved when the crystals are pumped by near-IR pulses centered between $1.1 \mu \mathrm{m}$ and $2 \mu \mathrm{m}$, typically from a near-IR optical parametric amplifier (OPA). $\mathrm{THz}$ pulses with $\mu \mathrm{J}$ energies have been demonstrated in such organic crystals [44]. Due to the higher center frequencies of the generated $\mathrm{THz}$ pulses compared to those from $\mathrm{LiNbO}_{3}$, the $\mathrm{THz}$ pulses can be focused to smaller diffraction-limited spots, thus resulting in electric fields on the order of tens of $\mathrm{MV} / \mathrm{cm}$ as demonstrated recently [22, 44]. Examples of intense THz pulses generated in organic crystals DSTMS and OH1 are shown in Fig. 2.
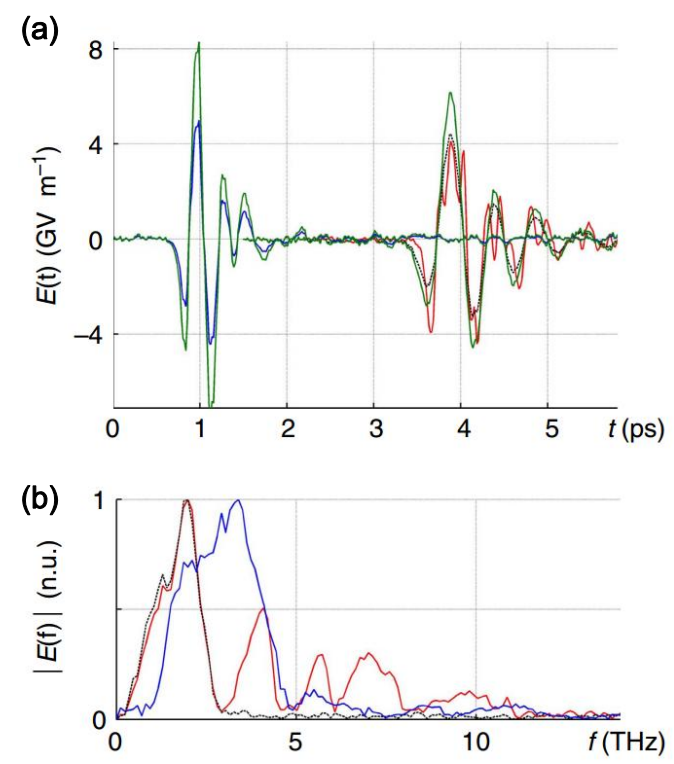

Fig. $2 \mathrm{THz}$ electric field profiles (a) and spectra (b) generated by OR in organic crystals DSTMS and OH1. (from [22])

In addition, gallium selenide ( $\mathrm{GaSe}$ ) is a nonlinear medium that allows the generation of multi-cycle $\mathrm{THz}$ radiation at higher frequencies (e.g., around $20 \mathrm{THz}$ ) by OR with collinear phase matching[27, 34]. These three $\mathrm{THz}$ generation methods are used in the works reviewed in this chapter. There are also other methods for strong and broadband THz pulse generation using either fs lasers in tabletop setups $[45,46]$ or relativistic electron bunches in accelerators $[47,48]$, which will not be discussed here. We note that the $\mathrm{THz}$ electric field profile is determined from the optical pump pulse intensity profile, so the $\mathrm{THz}$ fields used for $2 \mathrm{D} \mathrm{THz}$ spectroscopy are inherently carrier-envelope phasestable. This simplifies measurement of the full $\mathrm{THz}$ field as described below. 


\subsubsection{THz time-domain detection by electro-optic sampling}

THz pulses generated as described above typically have subpicosecond durations and can be sampled in the time domain using optical gate pulses of considerably shorter duration. For that purpose, a weak portion of the fs pulse used for THz generation is used to detect the $\mathrm{THz}$ electric field profile in a nonlinear optical crystal such as ZnTe and GaP via the electro-optic (EO) Pockels effect, i.e., electro-optic sampling (EOS) [49, 50]. As the $\mathrm{THz}$ generation and detection both originate from the same laser pulse, timing jitter between the gate and $\mathrm{THz}$ pulses is minimal. The same measurement method is used for the nonlinear $\mathrm{THz}$ signals in $2 \mathrm{D} \mathrm{THz}$ spectroscopy measurements. Because the timedependent $\mathrm{THz}$ electric field profile is measured in this manner, there is no need for heterodyne detection of the signal as typically used in 2D IR and visible spectroscopies.

The geometry for EOS is shown schematically in Fig. 3. The THz pulse and optical gate pulse, both with linear polarization, are focused into the EO crystal. Without the presence of the $\mathrm{THz}$ electric field, the gate pulse does not experience any birefringence in the EO crystal and remains linearly polarized. It is subsequently transmitted through a quarter wave-plate and becomes circular polarized. The horizontal and vertical polarization components $\left(I_{\mathrm{H}}\right.$ and $\left.I_{\mathrm{V}}\right)$ of the circularly polarized gate pulse are separated by a Wollaston prism (polarizing beamsplitter) and the difference (zero without the $\mathrm{THz}$ electric field) in their intensities is detected by a pair of balanced photodetectors. When the $\mathrm{THz}$ pulse is overlapped with the gate pulse in time, the $\mathrm{THz}$ electric field biases the EO crystal, causing a rotation $\Delta \phi$ of the index ellipsoid of the crystal and resulting in a transient birefringence. After transmission through the EO crystal and the quarter waveplate, the gate pulse becomes elliptically polarized. The difference between $I_{\mathrm{H}}$ and $I_{\mathrm{V}}$ is linearly proportional to the THz electric field in the limit of small $\Delta \phi$ and is measured by the balanced photodetectors. The polarity of the THz electric field determines the sign of $\Delta \phi$ and hence the sign of the detected signal. Sweeping the time delay $\Delta t$ between the $\mathrm{THz}$ pulse and the gate pulse, one can map out the electric field profile of the THz pulse. A numerical Fourier transformation of the $\mathrm{THz}$ field yields the complex $\mathrm{THz}$ spectrum.

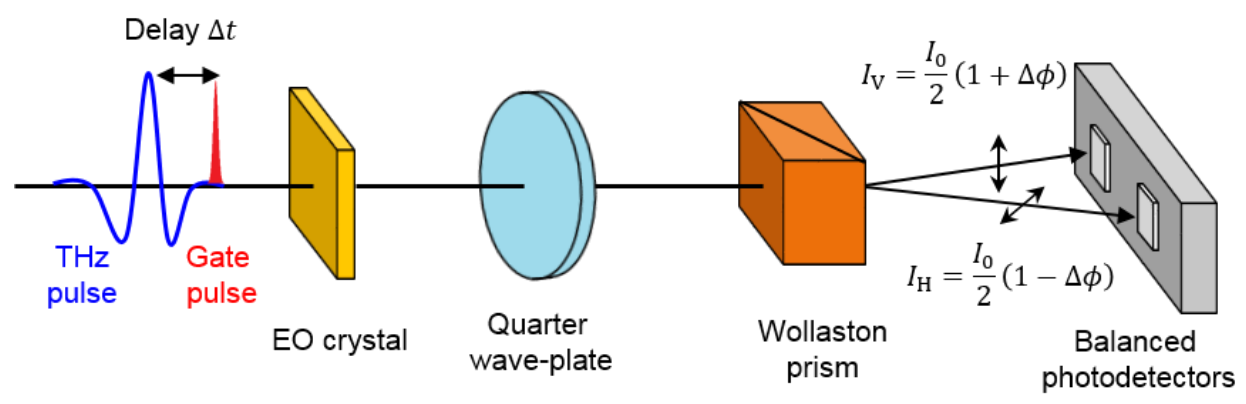

Fig. 3 Schematic illustration of the optical setup for $\mathrm{THz}$ electric field profile characterization via EOS. The phase retardation $\Delta \phi$ of the optical gate pulse in the EO 
crystal is converted to intensity modulations of the horizontal and vertical polarization components $I_{\mathrm{V}}$ and $I_{\mathrm{H}}$ by a quarter wave-plate and a Wollaston prism. The difference between $I_{\mathrm{V}}$ and $I_{\mathrm{H}}$ is detected by the balanced photodetectors. Sweeping the delay $\Delta t$ between the $\mathrm{THz}$ and gate pulses, the $\mathrm{THz}$ electric field waveform is mapped out.

In $\mathrm{THz}$ transmission measurements, the $\mathrm{THz}$ fields transmitted through the sample are collected and re-focused into the EO crystal for detection. If the sample under study has a resonant absorption in the $\mathrm{THz}$ excitation bandwidth, it absorbs and radiates $\mathrm{THz}$ radiation at its resonant frequency, manifested as a free-induction decay (FID) signal in the time domain. A numerical Fourier transformation of the FID signals yields the absorption or emission spectrum of the sample resonant with the excitation $\mathrm{THz}$ pulse. In a $2 \mathrm{D} \mathrm{THz}$ spectroscopy measurement, three $\mathrm{THz}$ field interactions with the sample produce the emitted $\mathrm{THz}$ field that is measured through EOS.

\subsubsection{Optical detection methods}

In many $\mathrm{THz}$ pump-probe experiments, a weak optical pulse is used as the probe pulse. It is time-delayed relative to and spatially overlapped at the sample with the THz pump pulse(s). The optical responses of the sample induced by the $\mathrm{THz}$ field, such as absorption [51], frequency shifts [52], optical harmonic generation [53], birefringence [54], and polarization rotation [55], are detected. Examples of $\mathrm{THz}$ pump-optical birefringence probe spectroscopy include the THz Kerr effect in liquid molecules [37, 54, 56], and ferroelectric crystals [57, 58], and THz-induced dipolar alignment of polar gas molecules [59, 60]. Here, $\mathrm{THz}$ electric fields orient or align the molecules and the orientational diffusion in the liquid or alignment revivals in the gas phase are monitored by optical birefringence through the anisotropic polarizability. Raman-active vibrational modes have also been observed using the THz pump-optical birefringence probe method, where anharmonic couplings between THz-driven THz-active modes and opticallydetected Raman-active modes are believed to play a role [37, 56, 58].

\subsection{Nonlinear 2D THz spectroscopy methods}

\subsubsection{Pulse sequences in 2D THz and 2D THz-Raman spectroscopies}

In 2D IR and visible spectroscopies, three pulses with controlled time delay between two neighboring pulses are usually used to conduct the experiments. In $2 \mathrm{D} \mathrm{THz}$ spectroscopy, there is only one example to date using three time-delayed $\mathrm{THz}$ pulses [33, 34]. This is limited in part by experimental difficulties in generation of multiple $\mathrm{THz}$ pulses and the recombination of the pulses at the sample. Most importantly, a very long data acquisition time is usually required, as all the inter-pulse delays and the time-domain signal detection time need to be scanned by mechanical delay stages. In this chapter, we mainly consider three types of pulse sequences and relevant light-matter interactions 
represented by the Feynman diagrams (details on the Feynman diagrams can be found in Refs. [1, 61, 62] etc.) shown in Fig. 4 which are typically used in different types of 2D $\mathrm{THz}$ spectroscopies. In the Feynman diagrams, the notations of the states may indicate the number of quanta in a particular degree of freedom in some cases, and in others they denote various levels of different modes. In the hybrid THz-Raman spectroscopies of liquid molecules, the transitions induced through $\mathrm{THz}$ or Raman excitations can in general include overtone and combination band transitions which are usually allowed in liquids where the intra- and intermolecular vibrations largely have strong anharmonicity[63].

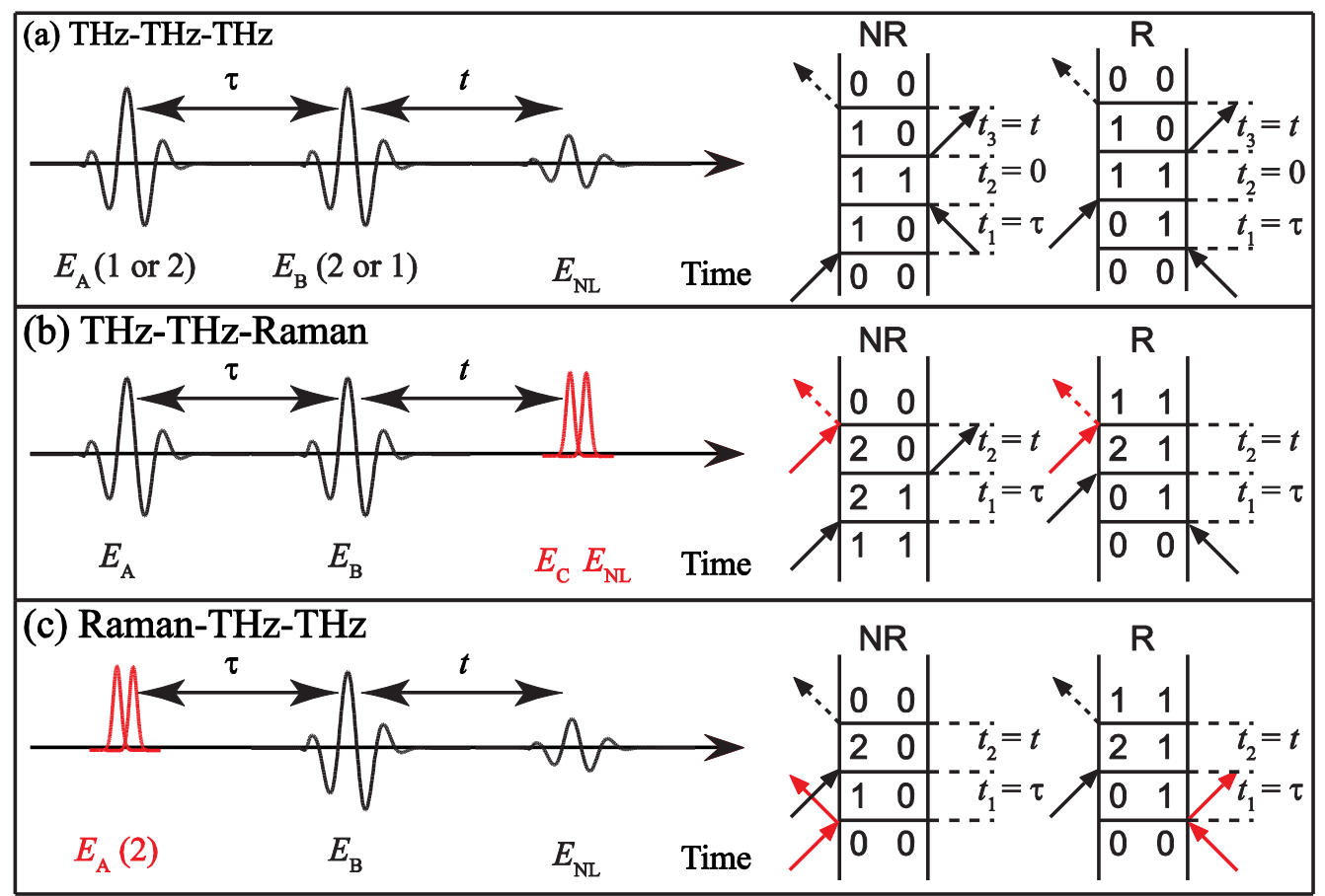

Fig. 4 Pulse sequences of 2D THz and 2D THz-Raman spectroscopies. The fields in black are $\mathrm{THz}$ fields and those in red are optical fields. Each field has one interaction with the sample unless indicated otherwise in parentheses. (a) THz-THz-THz sequence where all field-matter interactions arise from $\mathrm{THz}$ fields. The final signal emission is a THz field $E_{\mathrm{NL}}$ detected by EOS. Typical nonrephasing (NR) and rephasing (R) pathways following this sequence are described by the Feynman diagrams shown. (b) THz-THzRaman sequence where each $\mathrm{THz}$ field interacts with the sample once and a final Raman (optical) field interacts with the sample once $\left(E_{C}\right)$ to generate the nonlinear signal field $E_{\mathrm{NL}}$, which is detected optically through heterodyne mixing with the Raman pulse field. Typical NR and R pathways following this sequence are described by the Feynman diagrams shown. (c) Raman-THz-THz sequence where field-matter interactions involve a second-order Raman interaction by $E_{A}$ and a $\mathrm{THz}$ interaction by $E_{B}$. The final signal emission is a THz field $E_{\mathrm{NL}}$, detected by EOS. Typical NR and R pathways following this sequence are described by the Feynman diagrams shown. 
In the THz-THz-THz sequence shown in Fig. 4(a), all field-matter interactions involve THz fields. An example of the $\chi^{(3)}$ interaction pathways following the pulse sequence is illustrated here with examples of typical nonrephasing (NR) and rephasing (R) pathways shown by the Feynman diagrams. As shown in Fig. 4(a), THz field $E_{A}$ generates a firstorder coherence which evolves during time period $\tau$. THz field $E_{B}$ interacts with the sample twice, generating in succession a second-order population and a third-order coherence. The third-order coherence evolves during time period $t$ and radiates the signal, which is detected by EOS as a function of $t$. If we index the possible time delays by which field interaction they follow, then inter-pulse delay $\tau$ is the coherence time $t_{1}$; population time $t_{2}=0$ (field interactions 2 and 3 are time-coincident); and the detection time $t$ corresponds to $t_{3}$. $E_{A}$ can also interact twice to generate in succession a first-order coherence and a second-order population, the latter of which evolves during time period $\tau$. $E_{B}$ interacts once to generate a third-order coherence radiating the nonlinear signal. In this case, we have coherence time $t_{1}=0$ (first two interactions time-coincident) and population time $t_{2}=\tau$; the detection again, and generally, is $t=t_{3}$.

In the THz-THz-Raman sequence and typical Feynman diagrams shown in Fig. 4(b), THz fields $E_{A}$ and $E_{B}$ each interact with the sample once and together result in a secondorder coherence or population. Inter-pulse delay $\tau$ corresponds to the coherence time $t_{1}$ and time period $t$ corresponds to either a coherence time or a population time. The Raman pulse converts the second-order coherence or population into a Raman coherence which is detected optically. In birefringence detection, the detection time $t_{3}$ is integrated by the photodetector.

In the Raman-THz-THz sequence and typical Feynman diagrams shown in Fig. 4(c), a second-order interaction of the Raman pulse $E_{A}$ generates a Raman coherence which evolves during $\tau$. Via one THz interaction, THz pulse $E_{B}$ converts the Raman coherence into a second-order THz-active coherence radiating the signal field $E_{\mathrm{NL}}$, which is detected by EOS as a function of $t$. In this case, we have $t_{1}=\tau$ and $t_{2}=t$.

These three pulse sequences, as well as the pulse sequence in 2D Raman spectroscopy $[64,65]$, are complementary to each other. They allow one to study all THz-active modes, all Raman-active modes, or coupled THz- and Raman-active modes with flexibility. 


\subsubsection{Collinear phase matching and differential chopping detection}

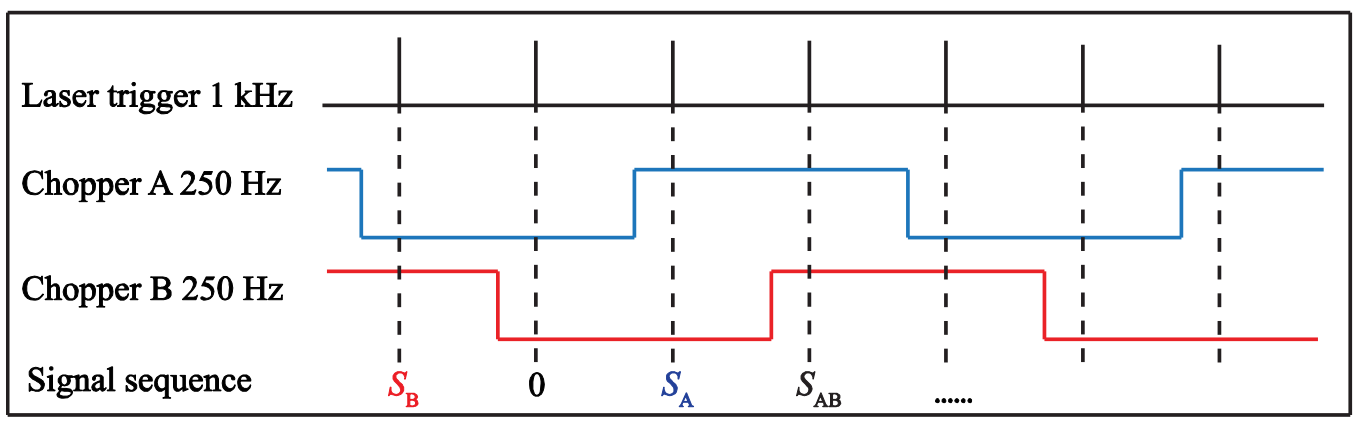

Fig. 5 Schematic representation of the differential chopping detection method.

As the wavelength of $\mathrm{THz}$ pulses is typically comparable to the spot size of a focused $\mathrm{THz}$ beam, the THz wavevector is not well-defined at the focus. The non-collinear FWM method with phase matching satisfied by the BOXCARS geometry, which has been routinely used in 2D IR and visible spectroscopies, cannot directly apply to $\mathrm{THz}$ fields. However, phase-resolved time-domain $\mathrm{THz}$ field detection and optical birefringence signal detection methods can allow nonlinear $\mathrm{THz}$ spectroscopy to be conducted with collinear phase matching, i.e., through wavevector-degenerate FWM.

To separate the nonlinear signals induced by both $\mathrm{THz}$ pulses from the signals induced by each THz pulse individually, a differential chopping detection method is usually used. Each $\mathrm{THz}$ pulse is modulated at a sub-harmonic frequency of the laser repetition rate by an optical chopper. One example of the differential chopping detection method is shown in Fig. 5. The laser repetition rate is $1 \mathrm{kHz}$. THz pulses $\mathrm{A}$ and $\mathrm{B}$ are both modulated at $250 \mathrm{~Hz}$. In four successive laser shots, one can detect the signal emerging from the sample in response to both pulses together, pulse A only and pulse B only, and no incident pulses (i.e., the background noise). The nonlinear signal field $S_{\mathrm{NL}}(t, \tau)$ that is measured as a function of inter-pulse delay $\tau$ and detection time $t$ is given by

$$
S_{\mathrm{NL}}(t, \tau)=S_{A B}(t, \tau)-S_{A}(t, \tau)-S_{B}(t),
$$

where $S_{A B}(t, \tau)$ is the signal field with both THz pulses present, and $S_{A}(t, \tau)$ and $S_{B}(t)$ the signal fields with either pulse A or B present individually. With collinear phase matching and the differential chopping detection method, the measured signal $S_{\mathrm{NL}}(t, \tau)$ is not restricted to $\chi^{(3)}$ signals, as nonlinear signals of other orders are also collinear. Numerical 2D Fourier transformation of $S_{\mathrm{NL}}(t, \tau)$ with respect to $t$ and $\tau$ yields the 2D spectrum as a function of detection and excitation frequencies denoted as $f$ and $v$, respectively. Different contributions to the signal field $S_{\mathrm{NL}}(t, \tau)$ associated with different interaction pathways have different characteristics and phase accumulation as functions of $t$ and $\tau$, so spectral peaks of different types can be separated in the 2D spectrum, which we will elaborate with examples in the subsequent sections. 
Differential chopping detection in experiments that have three separate $\mathrm{THz}$ pulses denoted by $\mathrm{A}, \mathrm{B}$ and $\mathrm{C}$ becomes more complicated, as the signals that originate from each individual pulse and each pair of pulses need to be accounted for. The nonlinear signal field is described by the following equation.

$$
\begin{aligned}
S_{\mathrm{NL}}\left(t, \tau, T_{\mathrm{w}}\right)= & S_{A B C}\left(t, \tau, T_{\mathrm{w}}\right)-S_{A B}\left(t, \tau, T_{\mathrm{w}}\right)-S_{B C}\left(t, T_{\mathrm{w}}\right)-S_{C A}\left(t, \tau, T_{\mathrm{w}}\right)+S_{A}\left(t, \tau, T_{\mathrm{w}}\right) \\
& +S_{B}\left(t, T_{\mathrm{w}}\right)+S_{C}(t),(5)
\end{aligned}
$$

where $\tau$ is the coherence time, $T_{w}$ is the population time or waiting time, and $t$ is the detection time. Details about the nonlinear signal detection method in experiments that involve three separate $\mathrm{THz}$ pulses can be found in references [33] and [34].

\subsection{Experimental setups for $2 \mathrm{D} \mathrm{THz}$ and $2 \mathrm{D}$ THz-Raman spectroscopies}

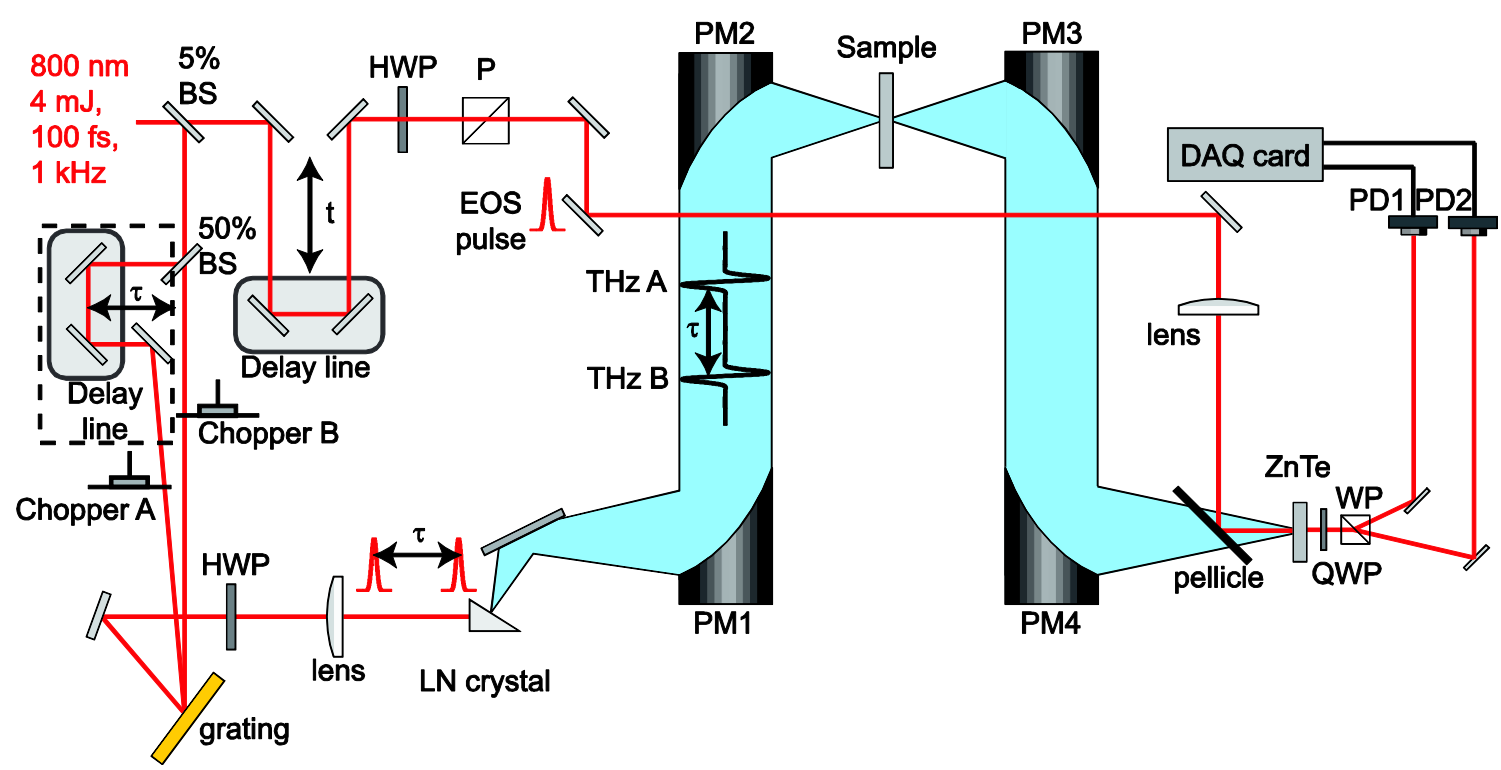

Fig. 6 An example of 2D THz spectroscopy experimental setup. Two time-delayed $\mathrm{THz}$ pulses are generated by two time-delayed optical pulses using the tilted-pulse-front method in a $\mathrm{LiNbO}_{3}$ crystal. The THz signals are detected by EOS in a ZnTe crystal. BS: beamsplitter, HWP: half wave-plate, QWP: quarter wave-plate, P: polarizer, WP: Wollaston prism, PM: parabolic mirror, LN: lithium niobate, PD: photodiode, DAQ card: data acquisition card. (from [31])

An example of the experimental setup for 2D THz spectroscopy is shown in Fig. 6 . Two time-delayed $\mathrm{THz}$ pulses are generated in a $\mathrm{LiNbO}_{3}$ crystal by OR of two timedelayed optical pulses recombined at the $\mathrm{LiNbO}_{3}$ crystal using the tilted-pulse-front method. The collinearly propagating $\mathrm{THz}$ pulses are collimated and focused into the sample by a pair of parabolic mirrors. The peak THz electric field strength is typically larger than $0.3 \mathrm{MV} / \mathrm{cm}$ for each pulse. $\mathrm{THz}$ signals transmitted through the sample are collected and re-focused into an EO crystal for detection. Two choppers are used to 
modulate the optical beams that generate the THz pulse pair. The differential nonlinear signal $E_{\mathrm{NL}}$ is processed by a data acquisition (DAQ) card. The total data acquisition time depending on the time windows required for $\tau$ and $t$ as well as data averaging typically ranges from one day to one week.

An example of the experimental setup with three separate $\mathrm{THz}$ pulses for $2 \mathrm{D} \mathrm{THz}$ spectroscopy is shown in Fig. 7. Three multi-cycle THz pulses centered at around $20 \mathrm{THz}$ which are time delayed with respect to each other are generated by OR in three separate $\mathrm{GaSe}$ crystals. Due to the relatively high frequency content of the pulses, the output $\mathrm{THz}$ radiation is nearly collimated with small divergence. The three $\mathrm{THz}$ beam paths are focused and recombined at the sample by one parabolic mirror. The resulting nonlinear signals are collected and focused onto a $\mathrm{ZnTe}$ crystal by a pair of parabolic mirrors. An ultrashort optical pulse from the laser oscillator is time-delayed and overlapped with the $\mathrm{THz}$ signals in a ZnTe crystal for high-bandwidth signal detection by EOS. Three choppers at 1/2,1/4 and 1/8 of the laser repetition rate are used for differential chopping detection.

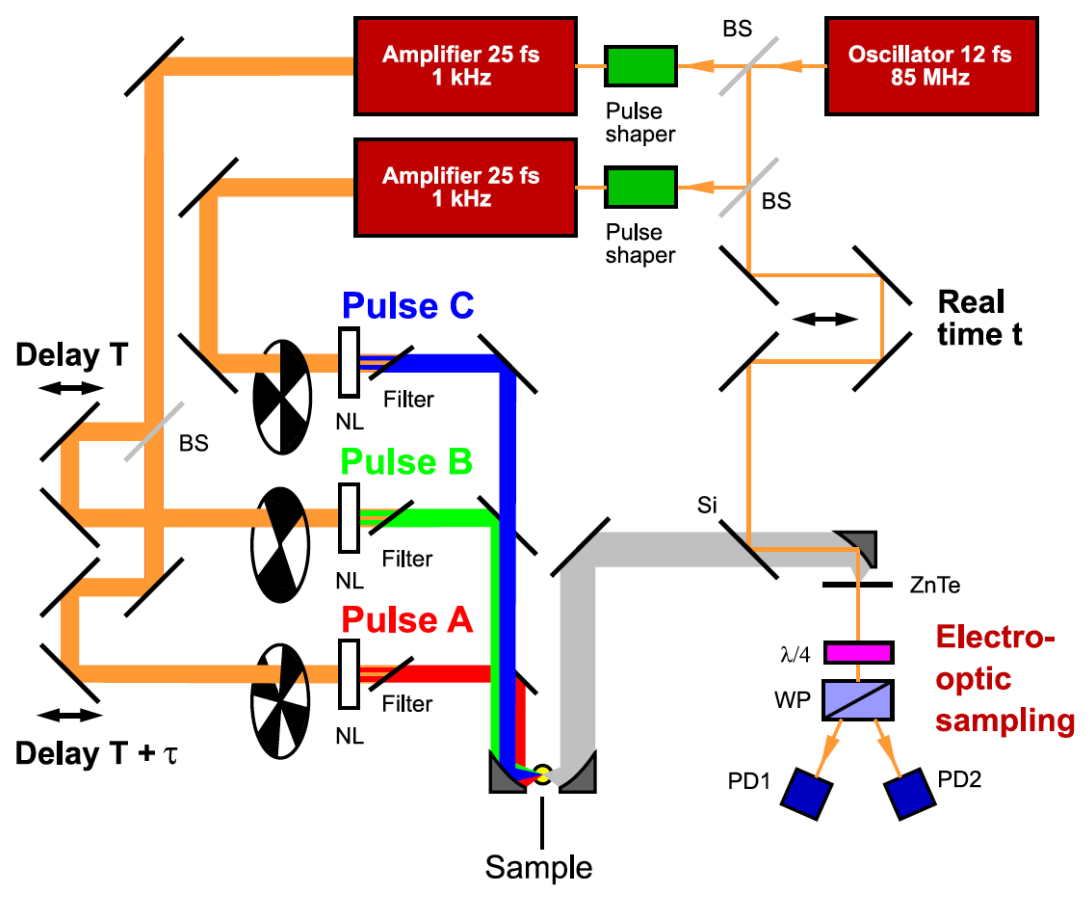

Fig. 7 An example of the experimental setup for $2 \mathrm{D} \mathrm{THz}$ spectroscopy with three separate $\mathrm{THz}$ pulses. (from [34]) 


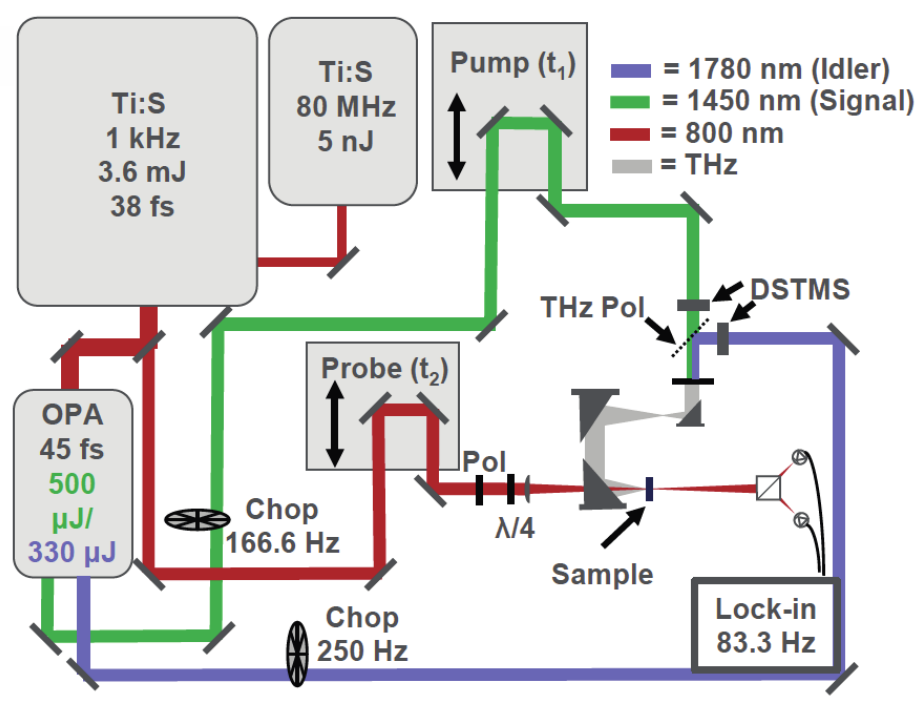

Fig. 8 An example of 2D THz-THz-Raman spectroscopy experimental setup. (from [38])

An example of the experimental setup for 2D THz-THz-Raman spectroscopy is shown in Fig. 8. Two DSTMS crystals are pumped by two time-delayed near-IR pulses from the signal and idler of an OPA. The time-delayed $\mathrm{THz}$ pulses, generated with orthogonal polarizations, are recombined by a $\mathrm{THz}$ polarizer and focused onto the sample by a pair of parabolic mirrors. A weak portion of the Ti:Sapphire laser pulse is time delayed with respect to the $\mathrm{THz}$ pulses and incident onto the sample through a hole in the parabolic mirror for birefringence measurements. Differential chopping detection is realized by chopping the signal and idler at $250 \mathrm{~Hz}$ and $166.6 \mathrm{~Hz}$, respectively, and detecting the differential signal at $83.3 \mathrm{~Hz}$ by a lock-in amplifier.

An example of the experimental setup for 2D Raman-THz-THz is shown in Fig. 9, which is essentially adapted from an optical pump-THz probe setup. Optical pulses from the Ti:Sapphire amplifier are split into three paths. One path is used for $\mathrm{THz}$ generation by $\mathrm{OR}$ in a GaP crystal. The generated $\mathrm{THz}$ pulses are focused onto the sample by an elliptical mirror. The THz signals transmitted through the sample are collected by another elliptical mirror and focused onto another GaP crystal for detection. The second portion of the optical pulse is time delayed with respect to the THz pulse and incident through a hole in the elliptical mirror onto the sample where it is used as a Raman pump. The third path is time delayed and recombined with the $\mathrm{THz}$ signal at the $\mathrm{GaP}$ crystal for $\mathrm{THz}$ detection by EOS. Differential chopping detection is realized similar to Fig. 6. The GaP crystals used for $\mathrm{THz}$ generation and detection provide broad bandwidth, but the $\mathrm{THz}$ field strength generated is not as strong as in the former two cases. As a result, a high repetition laser (5 kHz in Fig. 9), a sensitive detection scheme (Brewster windows and large numerical aperture optics shown in Fig. 9) and a long data averaging time (2 weeks in [39]) are required to observe weak nonlinear signals, such as the $\mathrm{THz}$ photon echo signals from water observed with this setup [39]. 


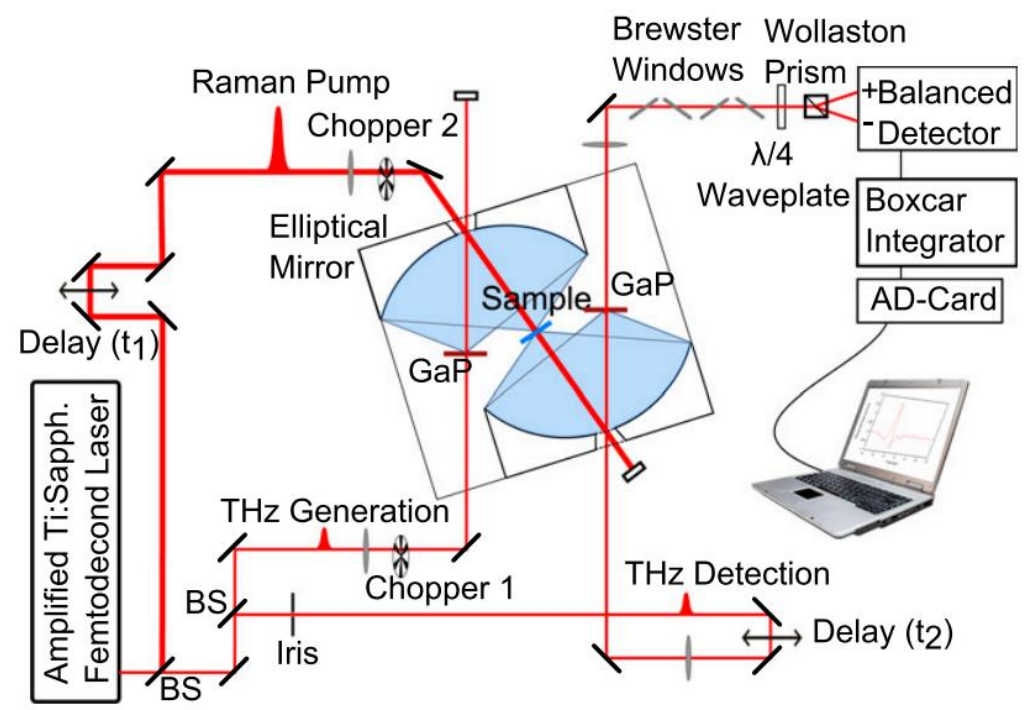

Fig. 9 An example of a 2D Raman-THz-THz spectroscopy experimental setup. (from [39])

\section{2D THz rotational spectroscopy of gas-phase molecules}

\subsection{Molecular orientation induced by THz pulses}

Rotational dynamics of polar molecules have been the subject of extensive efforts in coherent spectroscopy and coherent control. Motivated by interests in rotational angular momentum, energy relaxation processes, and high-order optical interactions with multilevel quantum systems, rotational excitations were examined by optical, microwave, and $\mathrm{THz}$ spectroscopies. Due to their unique energy level structure and their inherently quantum mechanical behavior, molecular rotations show dramatic differences from other degrees of freedom such as vibrations and spin precessions. We first discuss the linear responses of polar molecules to $\mathrm{THz}$ excitation. 
(a)
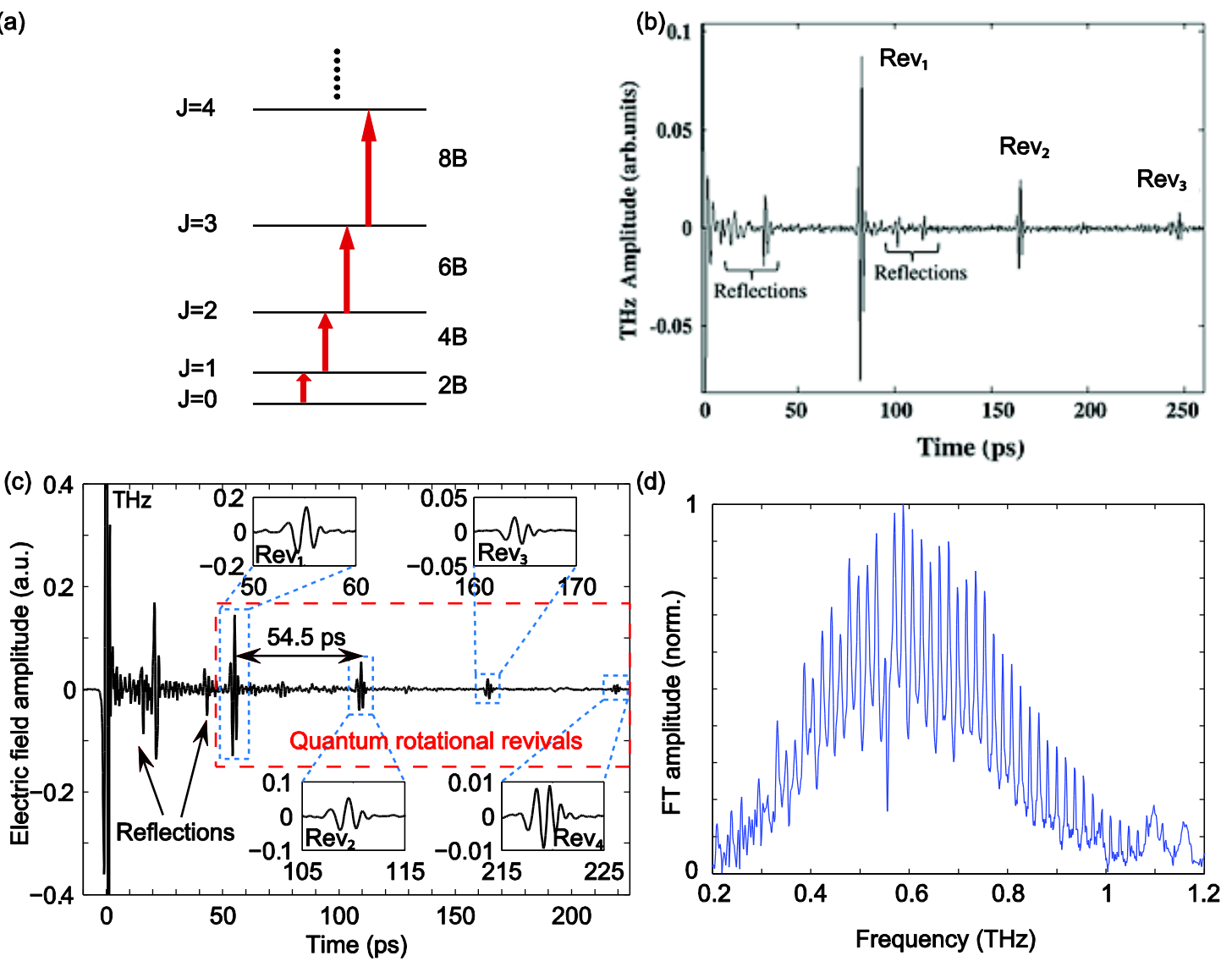

Fig. 10 (a) Schematic energy level diagram of molecular rotations within the rigid rotor framework. (b) and (c) FID signals from carbonyl sulfide (OCS) and acetonitrile $\left(\mathrm{CH}_{3} \mathrm{CN}\right)$ respectively. The superposition of all the rotational coherences results in bursts of $\mathrm{THz}$ emission called rotational revivals, labeled $\operatorname{Rev}_{1}, \operatorname{Rev}_{2}$, etc. (d) The rotational spectrum of $\mathrm{CH}_{3} \mathrm{CN}$ which results from a numerical Fourier transformation of the FID signals in (c) and which shows each rotational transition as a sharp peak. (from $[31,59]$ )

For small linear molecules with a permanent dipole moment, the Hamiltonian considering linear $\mathrm{THz}$ field-dipole interactions is given by

$$
H=H_{0}+H_{1}=\hat{J}^{2} / 2 I-\boldsymbol{\mu} \cdot \mathbf{E}_{\mathrm{THz}}(t) .
$$

The static Hamiltonian $H_{0}$ is assumed to be a rigid rotor Hamiltonian which accounts for the rotational dynamics of linear molecules. In $H_{0}, \hat{J}$ is the angular momentum operator and $I$ the moment of inertia, given by $I=h / 8 \pi^{2} c B$ where $h$ is the Planck constant, $c$ is the speed of light, and $B$ is the rotational constant of the molecule. $\widehat{H}_{0}$ in this form has an analytical solution. The eigenvectors are constructed by spherical harmonics and the eigenenergies are given by $E_{J}=2 h B c J(J+1)$ as shown in Fig. 10(a). Here, each eigenstate is denoted by the angular momentum quantum number $J$ and the energy difference between two adjacent $J$ states is given by $\Delta E_{J, J+1}=2 h B c(J+1)$. The fielddipole interaction Hamiltonian is $H_{1}=-\boldsymbol{\mu} \cdot \mathbf{E}_{\mathrm{THz}}(t)=-\mu E_{\mathrm{THz}}(t) \cos \theta$, where $\theta$ is the 
angle between the permanent dipole $\boldsymbol{\mu}$ and the polarization of $\mathbf{E}_{\mathrm{THz}}$. A THz field couples adjacent rotational states $J$ and $J+1$ (i.e. the selection rule is $\Delta J= \pm 1$ ) yielding 1 quantum coherences (1QCs) between adjacent states of the thermal ensemble. From the viewpoint of classical field-dipole interactions, the $\mathrm{THz}$ electric field exerts a torque on the dipoles, and the dipoles rotate at discrete $1 \mathrm{QC}$ frequencies $f_{J, J+1}=2 B c(J+1)$. The time-dependent $\mathrm{THz}$ emission from the rotating dipoles, given by the net dipolar orientation $\langle\cos \theta\rangle$, is the rotational free-induction decay (FID).

The THz pulse induces a net orientation of the molecular dipoles, i.e. nonzero $\langle\cos \theta\rangle$, but since the rotational coherences are at many different frequencies (determined by the different initial rotational levels $J$, many of which are thermally populated at ordinary temperatures), they rapidly go out of phase and the net orientation is lost. However, since the 1QC frequencies are all integer multiples of the lowest frequency $2 B c$, the dipoles go back in phase and there is a short-lived periodic "revival" of net dipole orientation $\langle\cos \theta\rangle$ with the quantum rotational revival period [66] given by $T_{\text {rev }}=(2 B c)^{-1}$. Upon each revival, the net dipolar orientation results in a macroscopic polarization in the sample, which emits a burst of coherent THz-frequency radiation. The FID signal thus consists of a sequence of such bursts separated by $T_{\text {rev }}$. Examples of the FID signals from carbonyl sulfide (OCS) [59] and acetonitrile $\left(\mathrm{CH}_{3} \mathrm{CN}\right)$ [31] gases excited by one single-cycle $\mathrm{THz}$ pulse generated from $\mathrm{LiNbO}_{3}$ and detected by EOS are shown in Figs. 10(b) and (c) respectively. The revival periods are $T_{\text {rev }}=82 \mathrm{ps}$ for OCS and $T_{\mathrm{rev}}=54.5 \mathrm{ps}$ for $\mathrm{CH}_{3} \mathrm{CN}$, both consistent with literature values of their rotational constants $B$. Fourier transformation of the periodic FID signal in Fig. 10(c) yields the linear rotational spectrum (the absorption spectrum) of $\mathrm{CH}_{3} \mathrm{CN}$ consisting of equally spaced peaks separated by $2 B C$ as shown in Fig. 10(d). Each rotational transition between adjacent rotational states is resolved as a sharp peak.

\subsection{Molecular alignment induced by THz pulses and two-quantum THz- THz-optical rotational spectroscopy}

The molecular orientational 1QCs discussed in Section 3.1 are the linear responses induced by one $\mathrm{THz}$ field-dipole interaction. Second-order nonlinear rotational responses resulting from two successive $\mathrm{THz}$ field-dipole interactions, manifested as 2-quantum coherences (2QCs) and non-thermal populations, have also been demonstrated. The second-order field-dipole interaction Hamiltonian takes the approximate form $\mathrm{H}_{2}=$

$\left(-\boldsymbol{\mu} \cdot \mathbf{E}_{\mathrm{THz}}(t)\right)^{2}=\mu^{2} E_{\mathrm{THz}}(t)^{2} \cos ^{2} \theta$, where we are neglecting other excitation pathways including stimulated scattering. 2QCs and excited rotational population correspond to net alignment of molecular dipoles, described by the alignment factor $\left\langle\cos ^{2} \theta\right\rangle$, with no net molecular orientation or associated macroscopic polarization because the dipoles may be antiparallel as well as parallel. Optical detection methods such as optical birefringence can be used to measure the alignment since it leads to anisotropy in the optical refractive 
indices between the direction of alignment ( $\mathrm{THz}$ electric field polarization direction) and the direction normal to the alignment direction. The experimentally measured birefringence is proportional to $\left\langle\cos ^{2} \theta\right\rangle$.
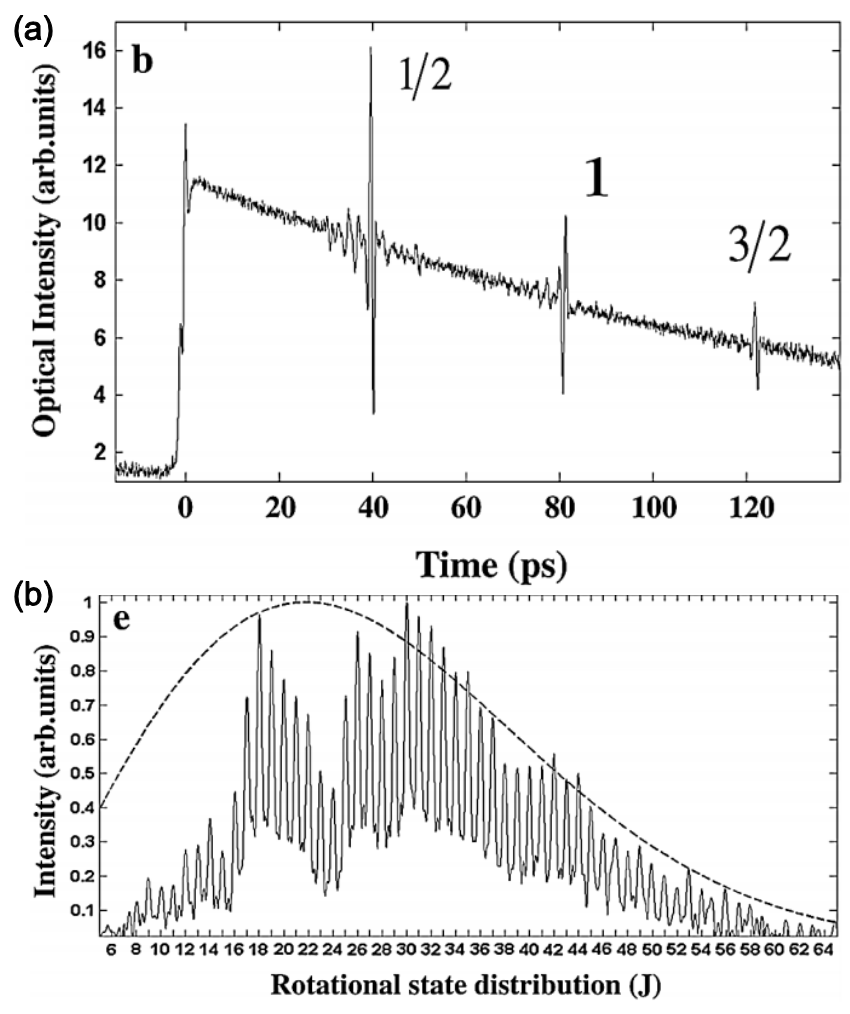

Fig. 11 (a) Alignment-induced birefringence signal from OCS. (b) Rotational spectrum of OCS resulting from a numerical Fourier transformation of the signal in (a). (from [59])

Rotational 2QCs result from two successive $\mathrm{THz}$ field-dipole interactions that generate coherent superpositions of first $J$ and $J+1$ levels and then $J, J+1$ and $J+2$ levels through successive transitions with the selection rule $\Delta J= \pm 1$. The 2QCs between levels $J$ and $J+2$ evolve in time at frequencies of $f_{J, J+2}=2 B c(2 J+3)$, and their superposition leads to periodic, short-lived revivals of constructive interference similar to those of the 1QCs but with a period of $T_{\mathrm{rev}} / 2=(4 B c)^{-1}$. The measurement results from OCS in Fig. 11(a) show that the 2QC revivals appear as expected. Fourier transformation of the birefringence signal yields the rotational spectrum in Fig. 11(b). Note that the successive resonant $\mathrm{THz}$ field interactions with the dipoles are completely different from non-resonant optical excitation of $2 \mathrm{QCs}$ through stimulated rotational Raman interactions with the molecular polarizabilities, with the selection rule $\Delta J= \pm 2$ [67]. 
(a)

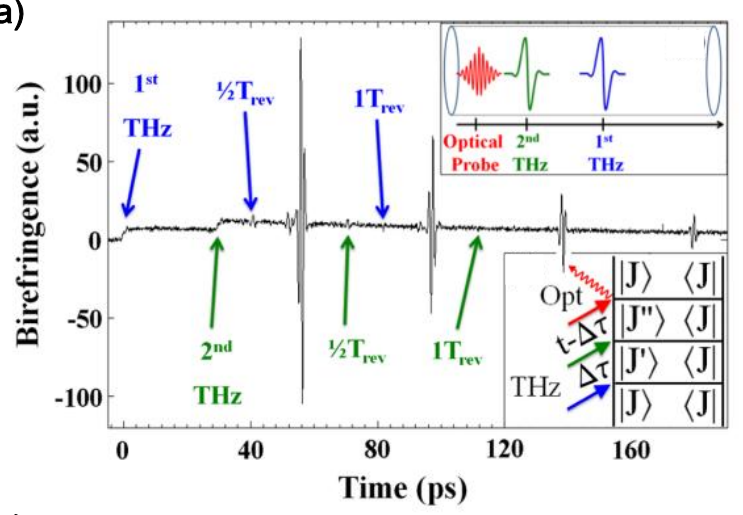

(b) $\quad \begin{array}{llllllll}10 & 20 & 30 & 40 & 50 & 60 & 70 p s\end{array}$

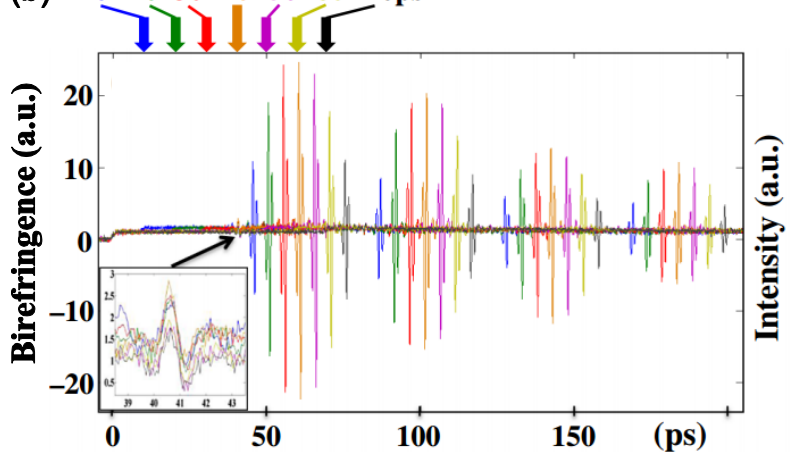

Fig. 12 (a) Optical birefringence signal from OCS in response to two THz pulses with a relative delay of 29 ps. Insets show the experimental geometry and the double-sided THzTHz-Raman Feynman diagram describing the 2QC excitation and detection pathway. (b) Optical birefringence signals from OCS in response to $\mathrm{THz}$ pulse pairs with variable relative delays color-coded as shown. The inset shows the far smaller birefringence signals induced by two successive interactions from one THz pulse. (from [60])

The periodic 2QC signals are superimposed on the steady-state second-order population response that is also induced by successive $\mathrm{THz}$ field interactions that produce first the 1QCs between adjacent levels $J$ and $J+1$ and then excited-state populations in levels $J+1$. The excited rotational populations are anisotropic because each thermally populated level $J$ includes equal populations in all of the $2 J+1$ degenerate rotational sublevels labeled by quantum number $M$, where $M=-J,-J+1, \ldots J-1, J$, while the excited population in level $J+1$ includes only the same sublevels and not the two sublevels $-(J+1)$ and $(J+1)$ because of the $\Delta M=0$ selection rule.

As the molecular alignment involves two $\mathrm{THz}$ field-dipole interactions, one can separate these two interactions and control the amplitudes of 2QCs using two timedelayed $\mathrm{THz}$ pulses. Molecular alignment in response to two time-delayed $\mathrm{THz}$ pulses has been measured [60], yielding the results shown in Fig. 12. The pulse sequence used in the experiment is shown in the inset of Fig. 12(a), which is the same as the THz-THzRaman sequence shown in Fig. 4(b). In Fig. 12(a), the birefringence signal induced by two $\mathrm{THz}$ pulses with an inter-pulse delay of $\tau$ is separated from the second $\mathrm{THz}$ pulse by 
$\tau / 2$. The $2 \mathrm{QC}$ signal level from time-delayed field-dipole interactions is far higher than that induced by two field-dipole interactions from either individual $\mathrm{THz}$ pulse. In Fig. 12(b), the birefringence signals are measured at several different inter-pulse delays and the 2QCs signals achieve the maximum amplitude when the delay $\tau$ is close to $T_{\text {rev }} / 2=$ $41 \mathrm{ps}$. This is because at $\tau=T_{\text {rev }} / 2$, the $1 \mathrm{QCs}$ induced by the first THz pulse with oddnumber $J$ are in phase with each other, and those with even-number $J$ are also in phase with each other, and, the alignment factor of the molecular dipoles is maximized. After the interaction with the second THz field, the generated 2QCs are in phase at $t=T_{\text {rev }} / 4$ $\left(T_{\text {rev }} / 4\right.$ after the second $\mathrm{THz}$ pulse). The constructive interference of the 2QCs is optimal at $\tau=T_{\mathrm{rev}} / 2$, which leads to the maximum coherent enhancement of the 2QC signal amplitudes [60].

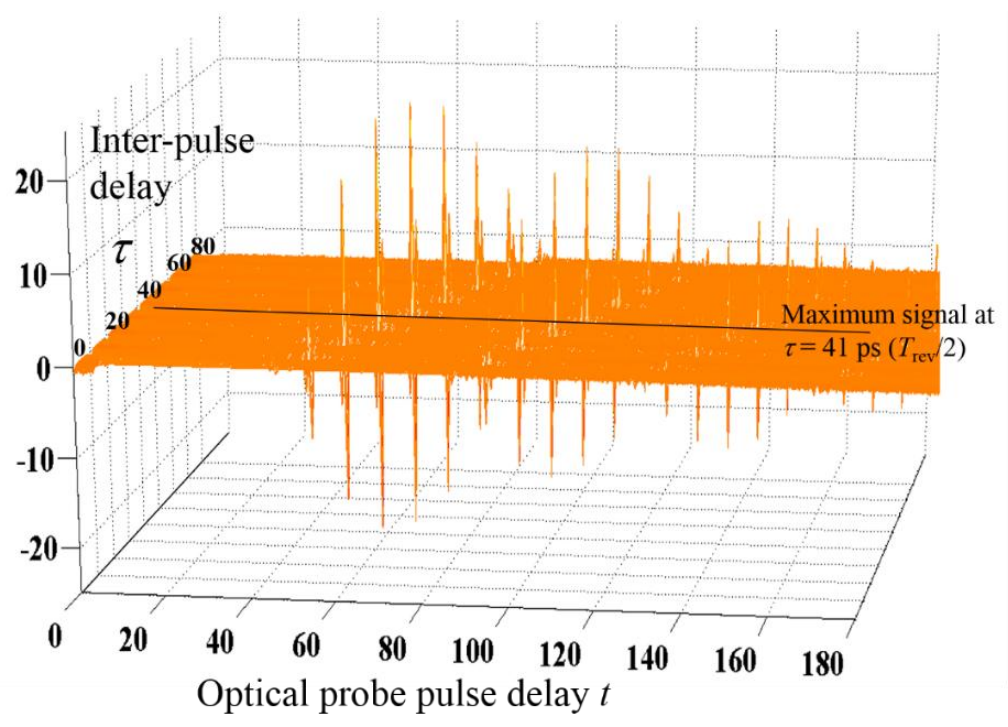

Fig. 13 2D time-domain plot of the optical birefringence signals from OCS in response to THz pulse pair.

This experiment demonstrates 2D two-quantum rotational spectroscopy in the time domain. In Fig. 13, we show the two-quantum signals at different time delays in a 2D plot which clearly shows that the maximum signal is achieved at $\tau=T_{\text {rev }} / 2$ (41 ps for OCS). 2D Fourier transformation of the 2D time-domain signal with respect to the interpulse delay $\tau$ and the detection time $t$ would yield a 2D rotational spectrum where 2quantum (2Q) signals appear at $J$-resolved positions along the frequency diagonal of $v=f / 2$, where the excitation and detection frequencies $v$ and $f$ are conjugate to the inter-pulse delay $\tau$ and the detection time $t$. This is because the phase accumulation of the 2QCs during $t$ is approximately twice as fast as the phase accumulation of the 1QCs during $\tau$. 


\section{3 $2 \mathrm{D} \mathrm{THz}$ rotational spectroscopy of acetonitrile}

In molecular rotations, there have been theoretical works that predict the possibility to observe $\mathrm{THz}$ photon echoes using both classical calculation of the rigid-rotor angular distribution [68] and quantum mechanical calculation of the dipolar orientation [26], in response to pairs of time-delayed $\mathrm{THz}$ pulses. The characteristic frequency patterns and the narrow linewidths typically observed in molecular rotational spectroscopy make it an ideal testbed for measurement of rephasing ( $\mathrm{R}$, or photon echo) and other contributions to 2D THz spectra. 2D THz rotational spectra were observed for the first time in $\mathrm{CH}_{3} \mathrm{CN}$ [31].

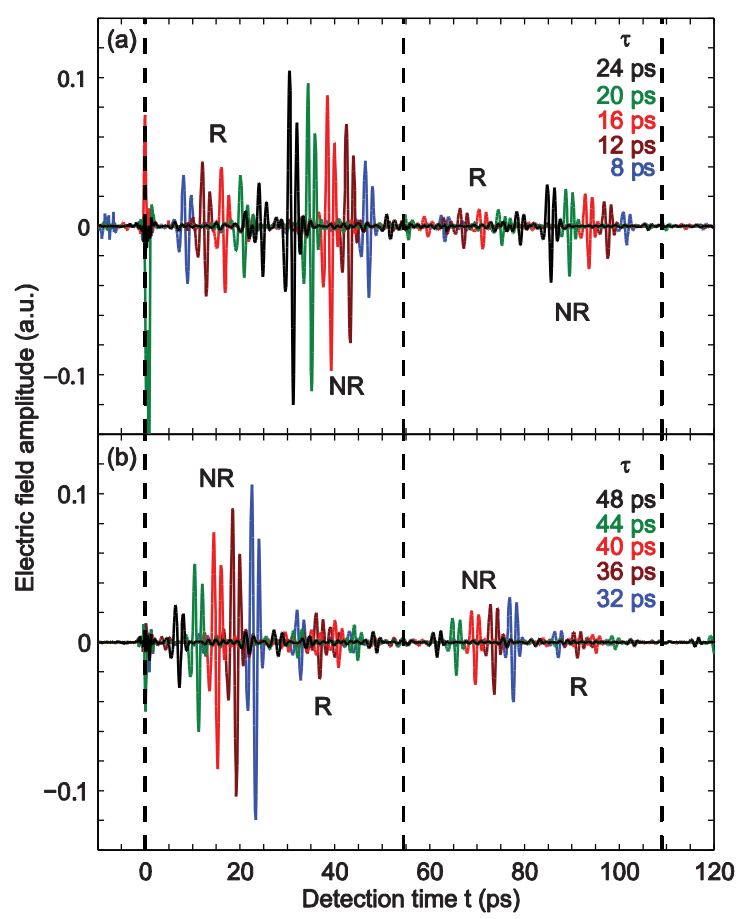

Fig. 14 (a) and (b) Experimental nonlinear time-domain signals $E_{N L}$ showing photon echoes (R) and non-rephasing signals (NR) at various inter-pulse delays $\tau$ indicated in the inset. The dashed lines are separated by $T_{r e v}=54.5$ ps. (from [31]) 


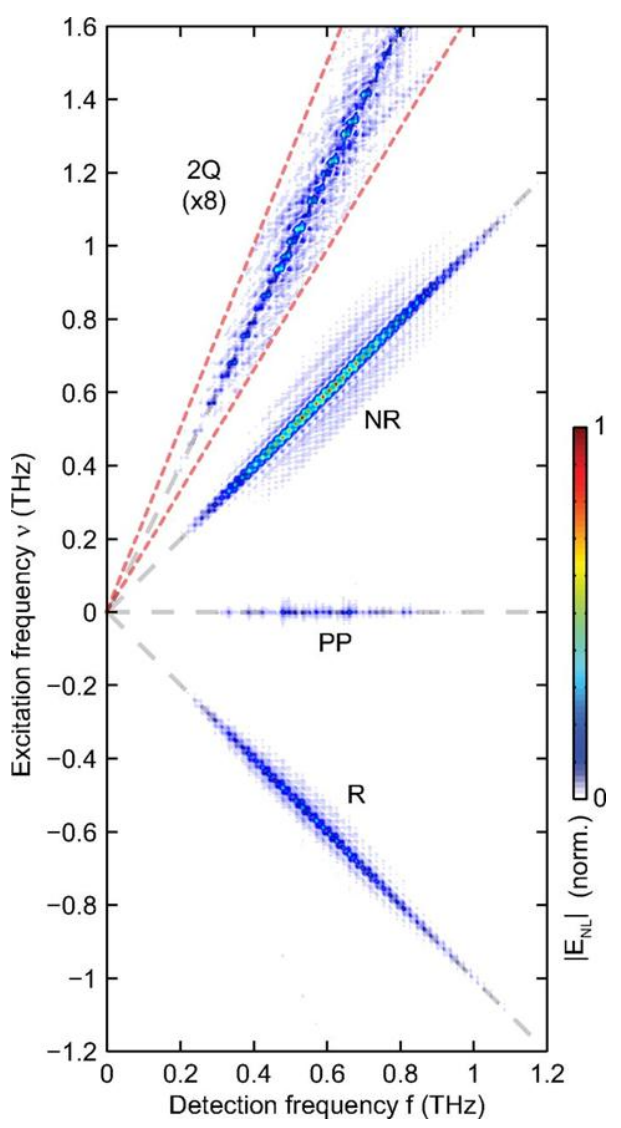

Fig. 15 Normalized $2 \mathrm{D} \mathrm{THz}$ rotational spectrum of $\mathrm{CH}_{3} \mathrm{CN}$. NR: nonrephasing, R: rephasing, PP: pump-probe, 2Q: 2-quantum, magnified $8 \times$. (from [31])

The experimental setup is the same as shown in Fig. 6, with a static pressure gas cell with $\mathrm{CH}_{3} \mathrm{CN}$ is placed at the sample position. Nonlinear signal traces $E_{\mathrm{NL}}$ as a function of EOS detection time $t$ at various inter-pulse delays $\tau$ are measured by the differential chopping detection technique. The nonlinear signals $E_{\mathrm{NL}}$ are shown in Fig. 14. THz pulse $\mathrm{B}$ is fixed at $t=0$, and a positive inter-pulse delay $\tau$ is incremented, which means $\mathrm{THz}$ pulse A appears earlier than pulse B. At each delay $\tau$ shown in the insets, a burst of $\mathrm{THz}$ signal appears at $t=\tau$, which confirms that the observed signals are photon echoes. As $\mathrm{THz}$ signal emission results from the collective polarization formed during the orientation of the molecular dipoles, the photon echo signals also show periodic revivals, of the same form as the linear FID signals. In this process, pulse A interacts with the dipoles once and generates first-order 1QCs which evolve and accumulate phase during $\tau$. Pulse B interacts with the dipoles twice, generating second-order populations and third-order 1QCs with reversed phase accumulation with respect to the first-order 1QCs. The thirdorder 1QCs are in phase at $t=\tau$ and at $t=\tau+n T_{\text {rev }}(n=1,2,3 \ldots)$, at which times they form collective polarizations that radiate the photon echo signals. At the revivals of $\mathrm{THz}$ pulse A, additional nonlinear signals are observed which are due to field-induced changes in the amplitudes of the revival signals generated by pulse A. These are 
identified as nonrephasing (NR) signals. In this signal pathway, the first two interactions are the same as those in the R pathway, and the second interaction with pulse B results in third-order 1QCs that are not phase-reversed with respect to the first-order 1QCs. The NR third-order 1QCs are hence in phase at the same time as the FID revivals induced by pulse A.

The 2D THz rotational spectrum $E_{\mathrm{NL}}(f, v)$ of $\mathrm{CH}_{3} \mathrm{CN}$ results from $2 \mathrm{D}$ numerical Fourier transformation of the nonlinear trace $E_{\mathrm{NL}}(t, \tau)$ recorded by measurement of $t$ dependent EOS signals at each inter-pulse delay $\tau$. The 2D magnitude spectrum is shown in Fig. 15. The spectrum is separated into the NR and R quadrants with a difference in the sign of the excitation frequency $v$. The NR quadrant consists of NR and 2Q signals which do not have reversed phase during time periods $\tau$ and $t$, while the $\mathrm{R}$ quadrant consists of $\mathrm{R}$ and also weaker fifth-order 2-quantum rephasing (2Q-R) signals which have reversed phase during $\tau$ and $t$. Along $v=0$, there are pump-probe (PP) signals which do not have phase accumulation during $\tau$. Each type of signal consists of $J$-resolved spectral peaks located at the frequencies of rotational $1 \mathrm{QCs}$ or $2 \mathrm{QCs}$ as will be discussed below.

(a)

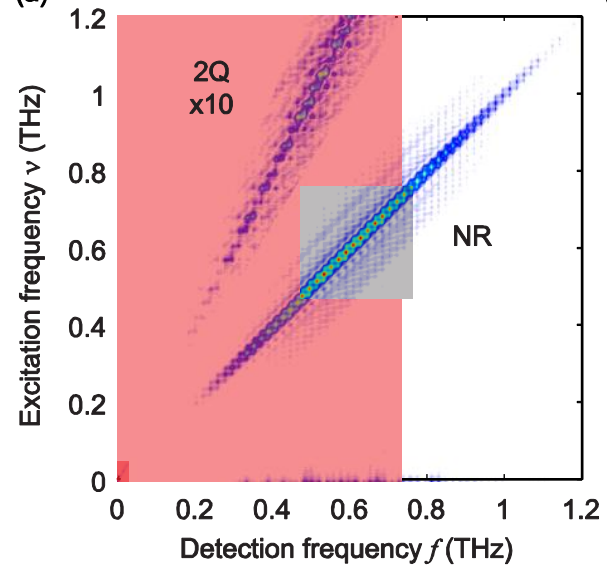

(b)

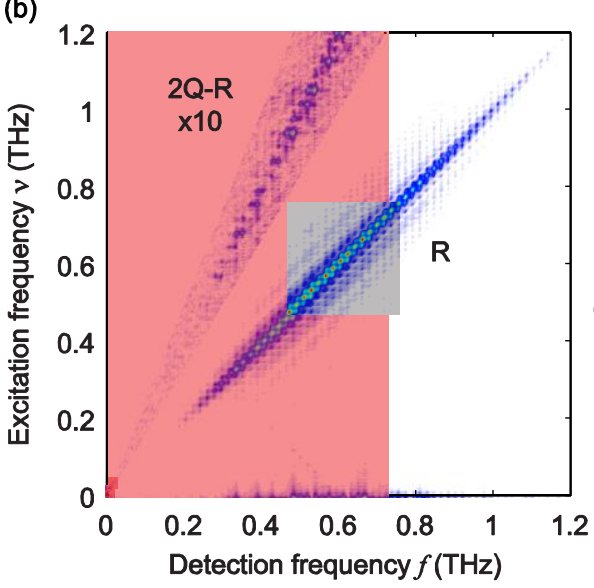

(c)

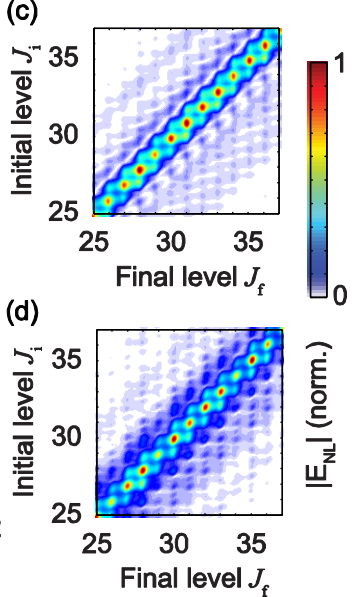

Fig. 16 (a) NR and (b) R (excitation frequency shown as positive) quadrants of the 2D spectrum of $\mathrm{CH}_{3} \mathrm{CN}$. Spectral amplitudes inside the red dashed area are magnified $10 \times$ to bring out the $2 \mathrm{Q}$ (in a) and 2Q-R (in b) signals. The dashed boxes cover rotational transition frequencies $f_{\mathrm{J}, \mathrm{J}}$, from $f_{25,26}$ to $f_{37,38}$. (c) and (d) Enlarged views of the NR (c) and R (d) spectra within the dashed boxes in the NR quadrant (in a) and R quadrant (in b) as functions of initial and final $J$ quantum numbers along the vertical and horizontal axes, respectively. Third- and fifth-order off-diagonal peaks are separated from the diagonal peaks at $J$-resolved positions. All of the spectra are normalized and plotted based on the color map shown. (from [31])

The NR and $\mathrm{R}$ quadrants of the 2D magnitude spectrum of $\mathrm{CH}_{3} \mathrm{CN}$ are plotted separately in Fig. 16, with the excitation frequency $v$ of the R quadrant made positive. 
Enlarged spectra at the centers of each quadrant are shown and plotted as a function of rotational $J$ quantum number. Each quadrant is normalized to its maximum amplitude. Weak spectral features of the fifth-order 2Q-R signals appear along the frequency diagonal $v=2 f$ when they are magnified. The THz field-dipole interaction pathways for the different types of signal are discussed as follows.

In the NR and R signals, $J$-state-resolved diagonal peaks are clearly observed along the frequency diagonals $v=f$ in Figs. 16(a) and 16(b). For these diagonal peaks, THz pulse A interacts once with the molecular dipoles to induce first-order 1QCs described by density matrix elements $|J\rangle\langle J+1|$. After inter-pulse delay $\tau$, pulse B interacts twice with the dipoles to induce in succession second-order rotational populations $|J+1\rangle\langle J+1|$ and third-order 1QCs $|J\rangle\langle J+1|$ (NR) or $|J+1\rangle\langle J|(\mathrm{R})$. The NR and R signals are radiated by these third-order 1QCs and are measured during the detection time $t$. The third-order nonlinear signal field $E_{\mathrm{NL}}(t, \tau)$ shows oscillations at the 1QC frequencies along the two time variables, and in this example the frequencies are the same, such that Fourier transformation of the signal with respect to both time variables yields $J$-state-resolved diagonal peaks at frequencies $v=f=f_{J, J+1}=2 B c(J+1)$ in the 2D spectrum. In addition, for each excitation frequency $v=f_{J, J+1}=2 B c(J+1)$, off-diagonal peaks are observed at detection frequencies $f=f_{J-1, J}=2 B c J$ and $f=f_{J+1, J+2}=2 B c(J+2)$. The off-diagonal peaks indicate that the first-order $|J\rangle\langle J+1|$ coherences induced by $\mathrm{THz}$ pulse A are correlated not only to the third-order coherences $|J\rangle\langle J+1|$ discussed above but also to the third-order coherences $|J-1\rangle\langle J|$ and $|J+1\rangle\langle J+2|$ involving two neighboring $J$ levels induced by pulse B. These spectral peaks are located at $J$-resolved positions as shown in the $2 \mathrm{D} J$-number map plotted as a function of initial and final rotational level $J_{\mathrm{i}}$ and $J_{\mathrm{f}}$ (related to frequencies variables by $v=2 B c\left(J_{\mathrm{i}}+1\right)$ and $\left.f=2 B c\left(J_{\mathrm{f}}+1\right)\right)$ in Figs. 16(c) and 16(d).

For 2Q and PP signals, pulse A interacts twice with the molecular dipoles to produce either $2 \mathrm{QCs}|J\rangle\langle J+2|$ or populations $|J+1\rangle\langle J+1|$ respectively. After inter-pulse delay $\tau$, pulse $\mathrm{B}$ interacts once with the dipoles to produce third-order $1 \mathrm{QCs}|J\rangle\langle J+1|$ that radiate the measured signals during time $t$. The $2 \mathrm{Q}$ signal field $E_{\mathrm{NL}}(\tau, t)$ shows oscillations as a function of $\tau$ at the 2QC frequencies and oscillations as a function of $t$ at the $1 \mathrm{QC}$ frequencies, giving rise to $J$-state-resolved peaks at $v \cong 2 f$. For PP signals, there is no coherence evolution during the inter-pulse delay so the signal appears in the 2D spectrum at zero frequency along $v$ and at $J$-resolved positions along $f$.

In addition to these third-order signals, fifth-order spectral peaks including 2Q-R signals and off-diagonal NR and R signals coupling $J$ and $J \pm 2$ levels are also observed. They originate from five $\mathrm{THz}$ field-dipole interactions, which are elaborated as follows. The 2Q-R peaks arise from two field interactions with pulse A to create 2QCs $|J\rangle\langle J+2|$ and three field interactions with pulse B. The first two interactions with pulse B create a population $|J+2\rangle\langle J+2|$ and the third induces a rephased $1 \mathrm{QC}|J+3\rangle\langle J+2|$ or 
$|J+2\rangle\langle J+1|$ which radiates the nonlinear signals during $t$. The $2 \mathrm{Q}-\mathrm{R}$ signals give rise to peaks along $f=2 v$ in the R quadrant shown in Fig. 16(b). For the fifth-order NR and R signals shown in Figs. 16(c) and 16(d), THz pulse A induces 1QCs $|J\rangle\langle J+1|$ evolving during $\tau$, and pulse B promotes them via four field-dipole interactions to fifth-order 1QCs with final rotational level, $J_{\mathrm{f}}$, two quanta away from the initial level $J_{\mathrm{i}}$ (namely, $\left|J_{\mathrm{f}}-J_{\mathrm{i}}\right|=$ $2)$ at $|J+3\rangle\langle J+2|(\mathrm{R})$ or $|J+2\rangle\langle J+3|(\mathrm{NR})$. The fifth-order 1QCs then radiate signals correlated to the $1 \mathrm{QCs}$ induced during $\tau$.

Example pathways of the $\mathrm{THz}$ field-dipole interactions described here are further elaborated by the double-sided Feynman diagrams presented in Fig. 17.

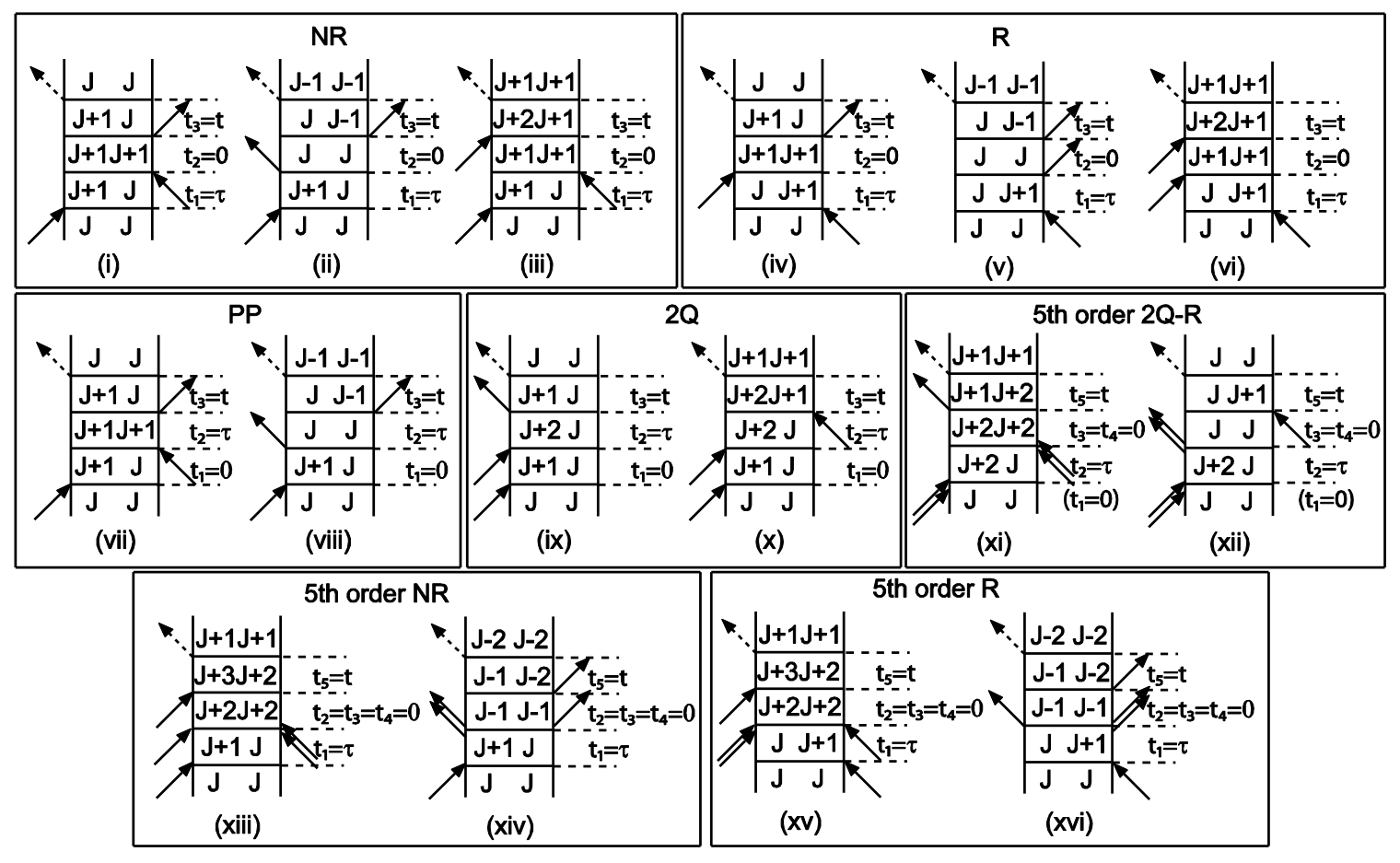

Fig. 17 Double-sided Feynman diagrams describing the THz field-dipole interactions. Diagram (i)-(iii) describes the third-order diagonal and off-diagonal peaks in the NR quadrant of the 2D spectrum. Diagrams (iv)-(vi) describe the third-order diagonal and off-diagonal peaks in the $\mathrm{R}$ quadrant. Diagrams (vii)-(viii) describe two excitation pathways that lead to the PP peaks. Diagrams (ix)-(xii) describe typical excitation pathways leading to the $2 \mathrm{Q}$ and $2 \mathrm{Q}-\mathrm{R}$ peaks. Diagrams (xii)-(xiv) and (xv)-(xvi) describe typical excitation pathways leading to the fifth-order NR and $\mathrm{R}$ off-diagonal peaks. The bra and ket symbols for the density matrix elements $|J\rangle\left\langle J^{\prime}\right|$ are assumed in all the diagrams. The time subscripts denote the number of preceding field interactions. The nonlinear signal emission time period $t$ corresponds to $t_{3}$ in all third-order processes and $t_{5}$ for the fifth-order processes. The inter-pulse delay time $\tau$ corresponds to $t_{1}$ for the $\mathrm{R}$ and NR signals (for third- and fifth-order signals) and to $t_{2}$ for the PP, 2Q and 2Q-R signals. (from [31]) 


\subsection{Extensions of $2 \mathrm{D} \mathrm{THz}$ rotational spectroscopy}

The initial demonstrations of $2 \mathrm{D} \mathrm{THz}$ rotational spectroscopy have provided illustrations of multiple-pulse $\mathrm{THz}$ coherent control over rotational dynamics and a detailed elaboration of the different signal contributions to 2D rotational spectra at third and fifth order. The results suggest possible experimental advances and a wide range of new information that can be uncovered. For example, by including a third $\mathrm{THz}$ pulse at a controlled delay with respect to the second pulse [33] such that the rotational population time can be varied, 2D rotational spectroscopy with three $\mathrm{THz}$ pulses can enable measurements of the time-dependent evolution of off-diagonal spectral peaks and spectral diffusion as in other 2D spectroscopies [69,70]. Such measurements may reveal specific rotational energy transfer and relaxation pathways (due to dipole-dipole interactions among the molecules under study, collisions with other species, etc.) by comparing the strengths and line shapes of the 2D spectral peaks for different population times. Independent control of the $\mathrm{THz}$ pulse polarizations could reveal the dynamics of relaxation among the $M$ sublevels of the rotational $J$ levels. The use of stronger $\mathrm{THz}$ pulses will allow measurement of still higher-order signal contributions, which will reveal additional correlations among rotational transitions and will allow the method to be used on molecules with moderate or small dipole moments. In addition to $\mathrm{THz}$ excitation pulses, optical excitation can be used to achieve enhanced control over rotational dynamics in linear and nonlinear molecules [71, 72]. Thus a wide range of experimental refinements is possible, offering prospects for new insights in to molecular rotational dynamics and the molecular interactions that mediate them.

\section{2D THz and hybrid 2D THz-Raman vibrational}

\section{spectroscopies}

Multidimensional IR vibrational spectroscopy has proved to be a powerful tool to study complex liquid-state vibrational dynamics, for example the hydrogen bonds in water $[5,73]$ and intramolecular vibrations in proteins and DNA [6, 7]. Multidimensional $\mathrm{THz}$ vibrational spectroscopy of liquids could extend the range to low-frequency molecular vibrations and intermolecular motions that may provide additional insights, for example, into the structural dynamics of water, proteins and DNA and to their chemical properties. However, 2D THz vibrational spectroscopy has so far been realized only on lattice vibrations in semiconductors. On the other hand, hybrid 2D spectroscopy combining $\mathrm{THz}$ and optical excitation or detection methods has enabled 2D THz-Raman variations that are complementary to $2 \mathrm{D} \mathrm{THz}$ and $2 \mathrm{D}$ Raman spectroscopies. As dipole and polarizability interactions are both involved, the hybrid methods can provide 
enhanced sensitivity to study some vibrational modes and their interactions. In this section, we discuss some of the 2D THz and 2D THz-Raman vibrational spectroscopies and their applications to the study of lattice vibrations in semiconductors, molecular vibrations in halogenated liquids and intermolecular dynamics of the hydrogen-bond networks in water and ionic aqueous solutions.

\section{1 $2 \mathrm{D} \mathrm{THz} \mathrm{spectroscopy} \mathrm{of} \mathrm{phonons} \mathrm{in} \mathrm{semiconductors}$}

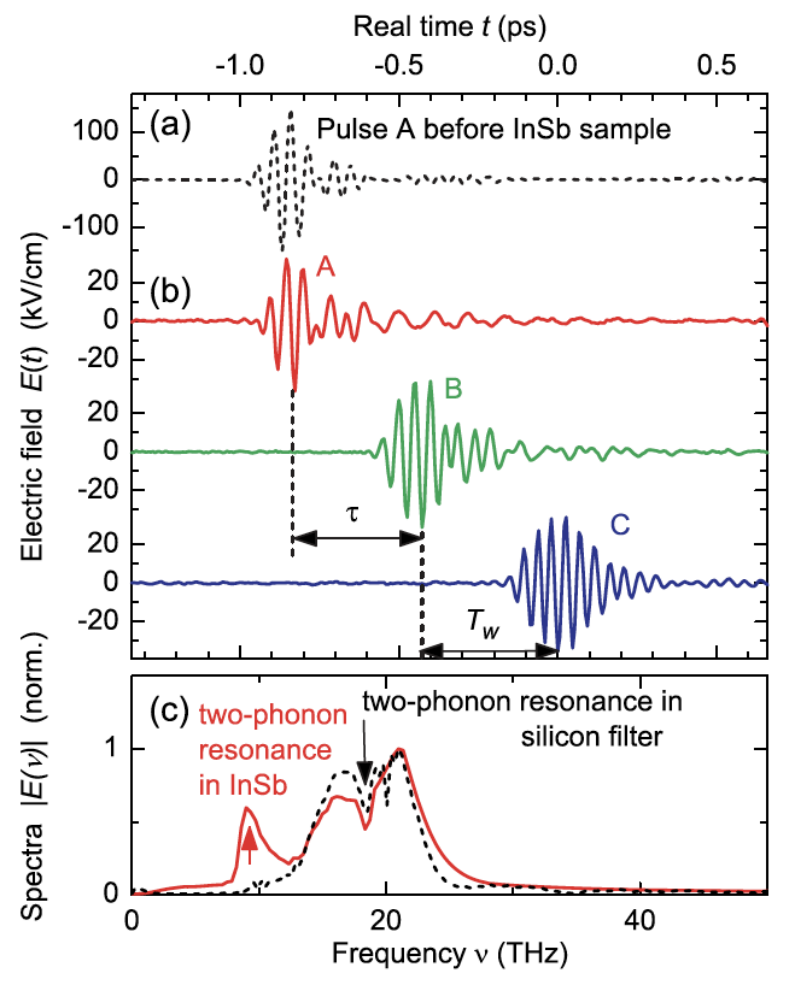

Fig. 18 (a) Waveform of $\mathrm{THz}$ pulse A before the InSb sample as a function of EOS detection ("real") time $t$. (b) Waveforms of pulses A, B, and C transmitted through the InSb sample. Each pulse develops oscillatory features emerging from the sample. The delays between pulses $\mathrm{A}$ and $\mathrm{B}$ and between pulses $\mathrm{B}$ and $\mathrm{C}$ are respectively the coherence time and waiting time denoted by $\tau$ and $T_{\mathrm{w}}$. (c) Fourier transform spectra of pulse A before the sample (dashed line) and transmitted through the sample (solid line). The dip at around $18 \mathrm{THz}$ is due to a two-phonon resonance in the silicon filter in the beam path, while the peak at around $10 \mathrm{THz}$ emerges from the two-phonon resonance in the InSb sample.

The first demonstration of $2 \mathrm{D} \mathrm{THz}$ spectroscopy with three $\mathrm{THz}$ pulses has been realized very recently in the study of phonon nonlinearity in the semiconductor indium antimonide (InSb) crystal $[33,34]$. The experimental setup is shown in Fig. 7. Multicycle $\mathrm{THz}$ pulses centered at $20 \mathrm{THz}$ with $6 \mathrm{THz}$ bandwidth are generated by OR in GaSe crystals and are incident onto the sample. The waveforms of the generated $\mathrm{THz}$ pulse and 
the three pulses transmitted through the sample denoted as A, B and C are shown in Fig. 18. Comparing the spectra of the incident $\mathrm{THz}$ pulse and the pulses transmitted through the sample, a spectral peak at $10 \mathrm{THz}$ emerges which is identified as a two-phonon coherence in InSb. As there is nearly no spectral amplitude at $10 \mathrm{THz}$ in the incident $\mathrm{THz}$ spectrum, the two-phonon coherence is generated nonresonantly by impulsive excitation.

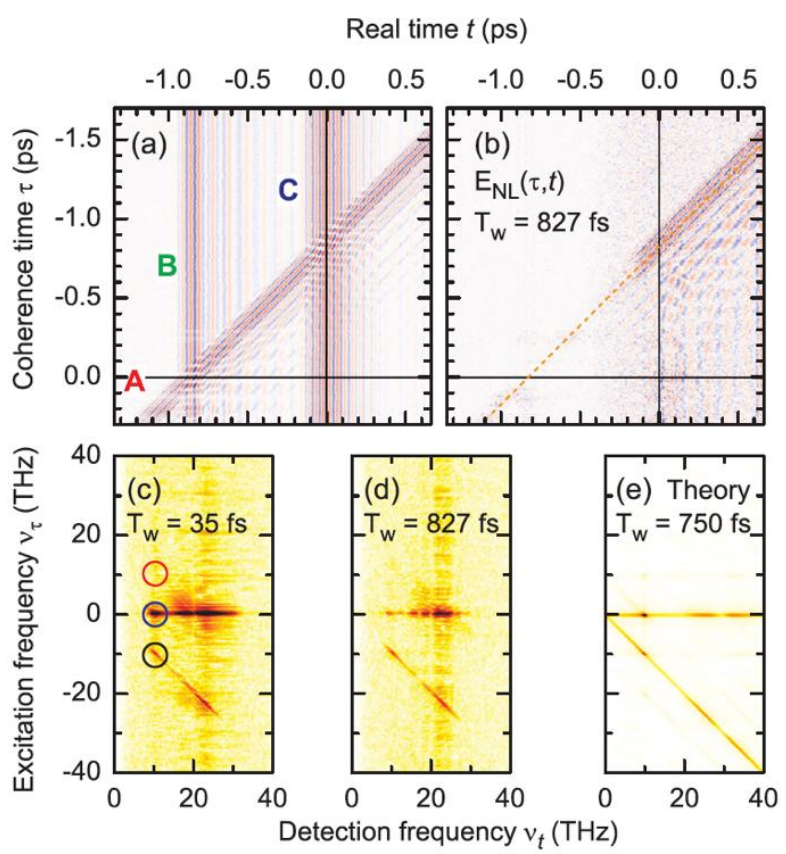

Fig. 19 (a) 2D plot of $S_{\mathrm{ABC}}\left(t, \tau, T_{\mathrm{w}}\right)$ which is superposition of the electric fields of all three pulses transmitted through the sample as a function of coherence time $\tau$ and real time $t$. (b) Nonlinear signal field $S_{\mathrm{NL}}\left(t, \tau, T_{\mathrm{w}}\right)$ extracted according to Eq. (6) for a waiting time $T_{\mathrm{w}}=827 \mathrm{fs}$. The dashed line indicates the center of pulse A. (c) and (d) 2D magnitude spectra $\left|S_{\mathrm{NL}}\left(v_{t}, v_{\tau}, T_{\mathrm{w}}\right)\right|$ for $T_{\mathrm{w}}=35$ and $827 \mathrm{fs}$. The circles in (c) mark the spectral peaks resulting from two-phonon resonances. (e) Calculated 2D spectrum for $T_{\mathrm{w}}=750 \mathrm{fs}$.

To study the nonlinear responses of the two-phonon coherences in detail, $2 \mathrm{D} \mathrm{THz}$ spectroscopy with three pulses was conducted. The pulse sequence is shown in Fig. 18. The coherence time and waiting (population) time are denoted by $\tau$ and $T_{w}$ respectively while the "real" time is the EOS detection time with its zero at the peak of pulse C. The 2D time-domain signals emerging from the sample in response to the three $\mathrm{THz}$ pulses and the 2D THz spectra at two different waiting times are shown in Fig. 19. The 2D timedomain data shown in Fig. 19(a) is the signal $S_{\mathrm{ABC}}\left(t, \tau, T_{\mathrm{w}}\right)$ in response to all three pulses. Pulses $\mathrm{B}$ and $\mathrm{C}$ have fixed relative delay, i.e. waiting time, $T_{\mathrm{w}}$, while pulse $\mathrm{A}$ is delayed with respect to pulse B. The data shown in Fig. 19(b) show the nonlinear signal $S_{\mathrm{NL}}\left(t, \tau, T_{\mathrm{w}}\right)$ extracted according to Eq. (6) at a selected waiting time of 827 fs. It is evident that there are oscillatory signals following the pulse that arrives latest (pulse A 
for $\tau<-850$ fs and pulse $\mathrm{C}$ for $\tau>-850 \mathrm{fs}$ ). The nonlinear signals exhibit interference patterns due to the presence of several signal contributions following different interaction pathways. 2D Fourier transformation of the signal in Fig. 19(b) gives rise to the 2D spectrum $S_{\mathrm{NL}}\left(v_{t}, v_{\tau}, T_{\mathrm{w}}\right)$ where $v_{t}$ and $v_{\tau}$ are detection and excitation frequencies at the selected waiting time $T_{\mathrm{w}}$. The experimental 2D magnitude spectra at $T_{\mathrm{w}}=35$ and $827 \mathrm{fs}$ and calculated 2D magnitude spectrum at a $T_{\mathrm{w}}=750$ fs are shown in Figs. 19 (c)-(e). There is very good agreement between the experimental data and calculation. The strong features at $v_{t}=22 \mathrm{THz}$ are mainly due to field-induced interband tunneling of carriers and interband two-photon absorption which are in the strongly nonperturbative regime due to the extremely large transition dipole between the valence and conduction bands [33]. The weak features at $v_{t}=10 \mathrm{THz}$ originate from the two-phonon resonances in $\mathrm{InSb}$, which are isolated from the 2D spectra and analyzed in the time domain in detail in the following.

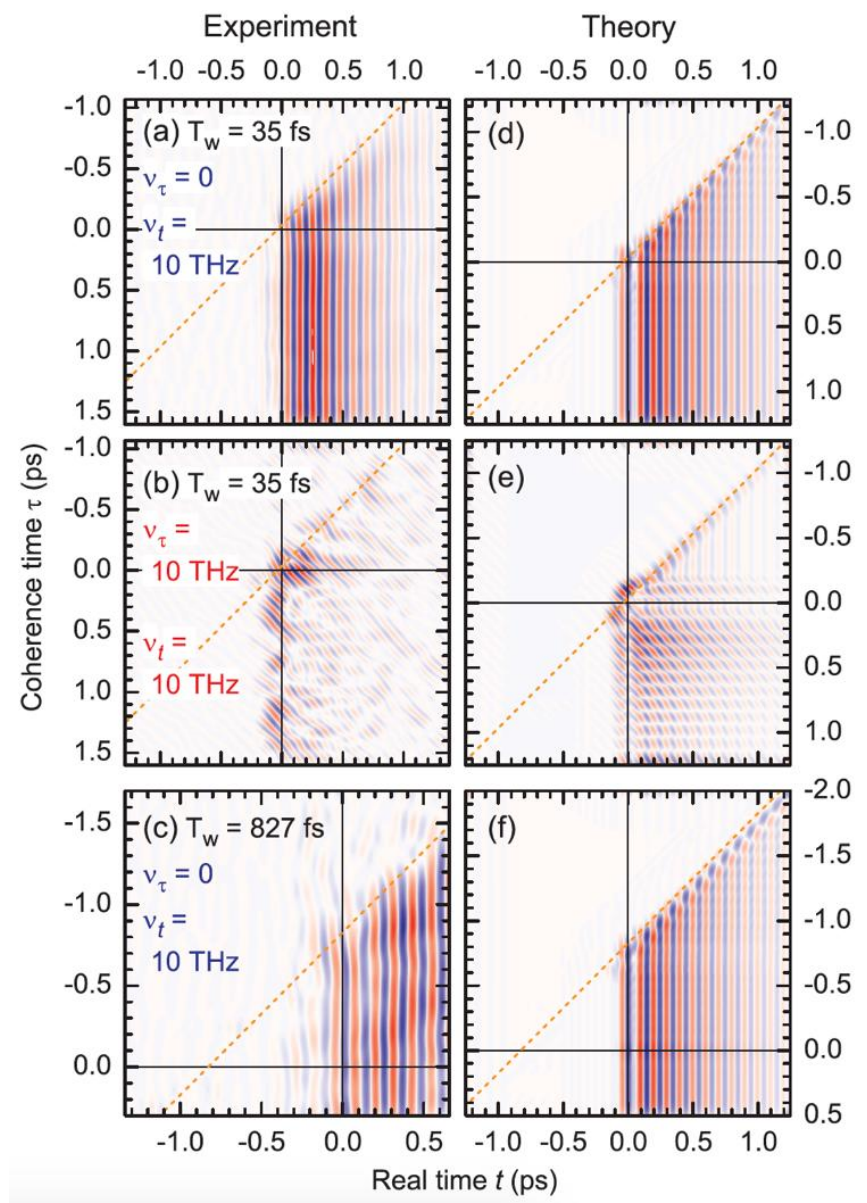

Fig. 20 Left column, experimental 2D time-domain signals from the spectral peaks at $\left(v_{\tau}, v_{t}, T_{\mathrm{w}}\right)=(0,10 \mathrm{THz}, 35 \mathrm{fs})(\mathrm{a})$, at $\left(v_{\tau}, v_{t}, T_{\mathrm{w}}\right)=(10 \mathrm{THz}, 10 \mathrm{THz}, 35 \mathrm{fs})(\mathrm{b})$, and at $\left(v_{\tau}, v_{t}, T_{\mathrm{w}}\right)=(0,10 \mathrm{THz}, 827 \mathrm{fs})$. Right column, calculated 2D time-domain signals resulting from the corresponding spectral peaks. The black solid lines mark the zeroes of coherence time and real time while the orange dashed line marks the center of pulse A. 
The 2D time-domain signals as functions of coherence time and real time resulting from inverse Fourier transformation of selected isolated spectral peaks in Figs. 19 (c)-(e) are shown in Fig. 20. There is good agreement between the experimental data and calculations. The signal in Fig. 20(b) is assigned as a rephasing signal as the phase front of the signal is perpendicular to the phase front of pulse A (orange dashed line), i.e., the nonlinear signal is phase-reversed with respect to the coherences generated by pulse A. Due to the lack of even-order signals in the data and the nonresonant nature of the twophonon coherence generation, the seventh-order $\left(\chi^{(7)}\right)$ pathway shown by the diagram (i) in Fig. 21 was proposed [33] as which is the lowest-order pathway to describe the lightmatter interactions. According to the diagrams, pulse A induces a two-phonon coherence via impulsive excitation with two interactions, and pulse B projects the two-phonon coherence back to the ground state also impulsively with two interactions. Finally pulse C generates a rephased two-phonon coherence of seventh order also via impulsive excitation, but with three interactions. This excitation process is shown separately in diagram (i). The two-phonon coherence radiates the nonlinear signal shown in Fig. 20(b).

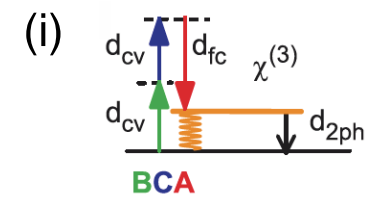

(ii)

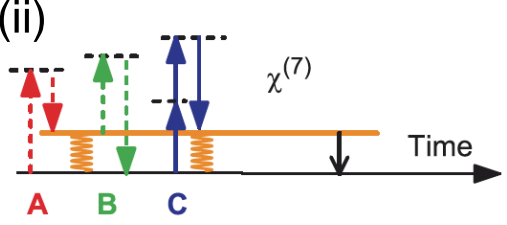

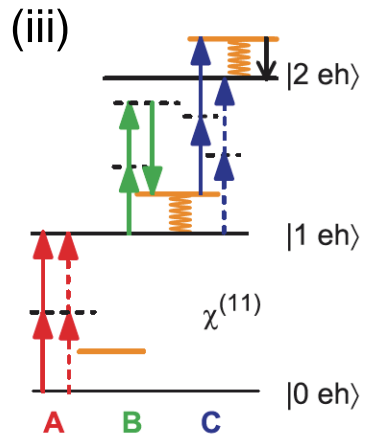

Fig. 21 Ladder diagrams describing relevant light-matter interactions for impulsive generation of two-phonon coherences (i), experimentally observed R signal (ii) and NR signal (iii). Time evolution is rightward. The red, green and blue solid and dashed arrows represent interactions between pulses A, B, and C and the system on the ket and bra sides respectively. The yellow lines represent two-phonon states and the yellow wavy lines represent two-phonon coherences. The black arrows pointing downward denote final nonlinear signal emission.

The origins of the nonlinear signals in Figs. 20(a) and 20(c) were assigned to an eleventh-order $\left(\chi^{(11)}\right)$ NR pathway which was proposed as the lowest-order pathway that can lead to these signals. It is described by the ladder diagram (iii) in Fig. 21 and analyzed as follows. Each pulse has to interact at least once to lead to the nonlinear signal. Due to the observation that the phase front of the signal is independent of that of pulse A, pulse A should interact an even number of times. A third-order process hence is not sufficient to explain the signal origin. As shown in Fig. 4 of reference [33], the nonlinear signal at $T_{\mathrm{w}}=827 \mathrm{fs}$ is found to be a direct continuation in amplitude and phase of that at $T_{\mathrm{w}}=35 \mathrm{fs}$. Besides, its phase is independent of the pulse sequences. Hence the 
nonlinear two-phonon coherence signal follows the phase of pulse B only, while pulses A and $\mathrm{C}$ create long-lived electronic excitations in InSb whose bandgap is about $41 \mathrm{THz}$, twice the frequency of the THz pulses. The ladder diagram (iii) shown in Fig. 21 can be read as follows. Pulse A excites an electronic population via four interactions, pulse B impulsively induces a two-phonon coherence via three interactions, pulse $\mathrm{C}$ promotes this two-phonon coherence from the first electronic excited state to the second one, and finally the two-phonon coherence at the second electronic excited state radiates the nonlinear signal.

In this experiment, the exceptionally high-order nonlinear interactions were proposed on the basis of the huge transition dipole moments associated with the electronic and vibronic transitions in the InSb sample. In pure vibrational systems where nonlinearity is not as dramatic, 2D spectra resulting from all $\mathrm{THz}$ interactions have so far not been available. Hybrid 2D THz-Raman methods can provide better sensitivity due to simultaneous interactions via dipole and polarizability. Several examples of $2 \mathrm{D} \mathrm{THz}-$ Raman vibrational spectroscopies are discussed in the following sections.

\subsection{D THz-THz-Raman spectroscopy of intramolecular vibrations in liquids}

A demonstration of 2D THz-THz-Raman spectroscopy has successfully revealed the anharmonic couplings between intramolecular vibrational modes in halogenated liquids [37]. The experimental setup used is the same as in Fig. 8. Two THz pulses with orthogonal polarizations and a relative time delay $\tau$ are generated from two DSTMS crystals and are focused into the sample to excite the liquid. An optical probe pulse that is delayed from the second $\mathrm{THz}$ pulse by time $t$ and polarized at $45^{\circ}$ with respect to each $\mathrm{THz}$ pulse is incident onto the sample to probe the THz-induced response. The birefringence of the probe pulse is measured as a function of $\mathrm{THz}$ inter-pulse delay $\tau$ and measurement time $t$.

Let us first consider the experiment with a single $\mathrm{THz}$ excitation pulse, i.e., a $\mathrm{THz}$ pump-optical birefringence probe measurement [56]. The excitation bandwidth spanning 1-5 $\mathrm{THz}$ includes $\mathrm{THz}$-active vibrational modes in many liquids. The transient birefringence of the probe pulse is measured as a function of the delay $t$ between the $\mathrm{THz}$ and optical pulses. The results are shown in Fig. 22. During the $\mathrm{THz}$ pulse, an instantaneous birefringence signal that scales with the square of the $\mathrm{THz}$ electric field emerges due to the $\mathrm{THz}$ nonresonant electronic Kerr effect $[54,56]$. A slow decay due to the THz-induced orientation of the molecules, i.e. the THz rotational Kerr effect, and subsequent diffusion to an isotropic distribution. On top of the orientational signal, oscillatory signals corresponding to Raman-active vibrational modes are observed. These 
Raman-active modes are excited via two $\mathrm{THz}$ interactions and the excitation pathways are revealed in 2D THz-THz-Raman spectroscopy as discussed below.
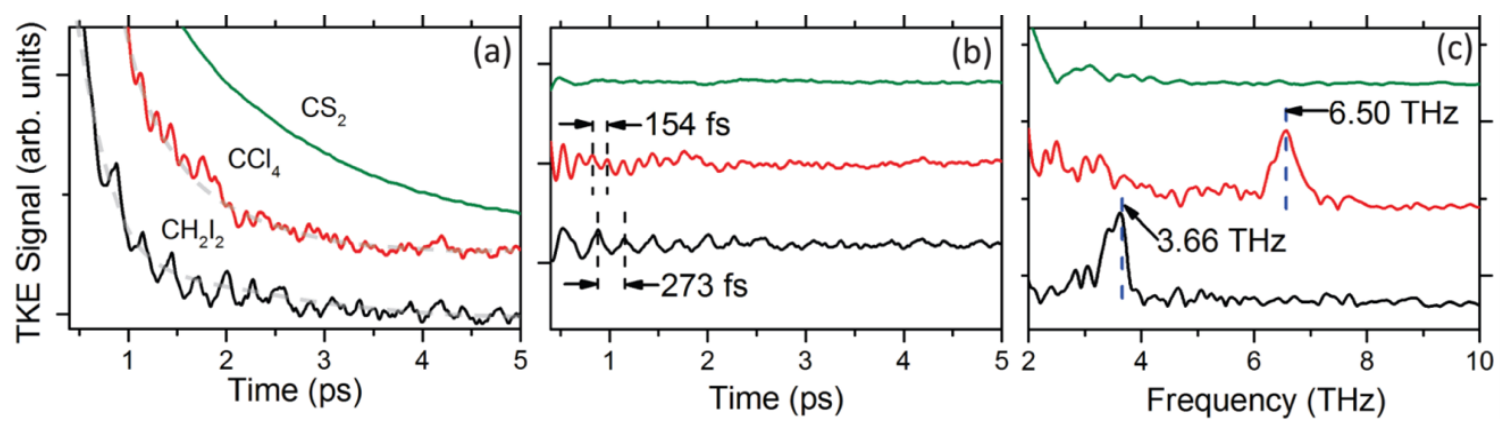

Fig. 22 (a) Orientational diffusion and vibrational coherences observed in $\mathrm{THz}$ Kerr effect measurements of halogenated liquids. (b) Vibrational signals extracted from (a). (c) Fourier transforms of the oscillatory signals in (b) reveal the vibrational spectra. (from [56])

In the 2D THz-THz-Raman spectroscopy experiment, differential chopping detection ensures that the nonlinear signal $S_{\mathrm{NL}}$ results from one interaction with each $\mathrm{THz}$ pulse, as the signals resulting from two interactions with either individual $\mathrm{THz}$ pulse are excluded. An example of the THz field-matter interaction pathways is illustrated following the THz-THz-Raman pulse sequence shown in Fig. 4(b). Let us consider a three-level system consisting of three states $|a\rangle,|b\rangle$, and $|c\rangle$ which denote the combination bands $|1,0\rangle$, $|0,1\rangle$, and $|2,0\rangle$ of two vibrational modes. The transitions between each pair of states can be both THz- and Raman-allowed. The typical pathways for different types of signals are described by the Feynman diagrams shown in Fig. 23. We elaborate one possible pathway as shown in diagram (i) describing the NR signals observed in Ref. [37] as follows. The system starts with a population $|1,0\rangle\langle 1,0|$. THz pulse A interacts once to generate a first-order coherence $|2,0\rangle\langle 0,1|$, which evolves at frequency $\omega_{b c}$ during the time period $\tau$ (or $t_{1}$ in [37]). THz pulse $\mathrm{B}$ interacts once to induce a second-order coherence $|2,0\rangle\langle 1,0|$ which evolves at frequency $\omega_{a c}$ during the time period $t$ (or $t_{2}$ in [37]). As this coherence is correlated to the amplitude of $|2,0\rangle\langle 0,1|$, the amplitude of $|2,0\rangle\langle 1,0|$ is modulated as the delay $\tau$ is varied. The optical probe pulse detects $|2,0\rangle\langle 1,0|$ at each $t$ point through a Raman transition between $|2,0\rangle$ and $|1,0\rangle$. The transient birefringence that the probe pulse experiences measures the amplitude of $|2,0\rangle\langle 1,0|$. The interaction pathway is described by a third-order response function $R^{(3)}(\tau, t)$ as follows:

$$
R^{(3)}(\tau, t) \propto \Pi_{c a} \mu_{b a} e^{-i \omega_{a c} t} \mu_{b c} e^{-i \omega_{b c} \tau} \rho_{\mathrm{eq}},
$$

where $\Pi_{x y}$ and $\mu_{x y}$ are the polarizability and dipole transition moments and $\rho_{\mathrm{eq}}$ is the density matrix at equilibrium. The 1D signals shown in Fig. 22 essentially arise from the same response function with $\tau$ set to zero. 2D Fourier transformation of the response 
function with respect to $\tau$ and $t$ gives rise to an off-diagonal peak located at $(f, v)=$ $\left(\omega_{a c}, \omega_{b c}\right) / 2 \pi$ showing the coupling between the two vibrational modes.

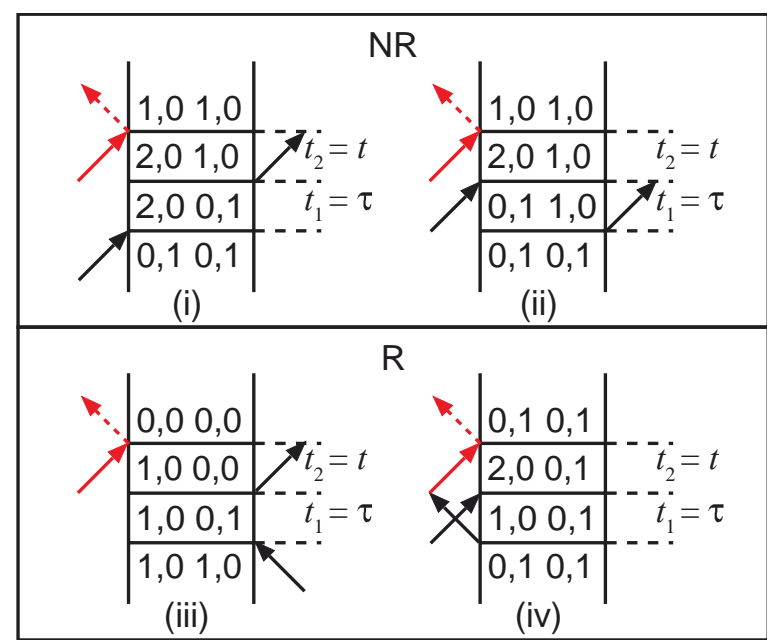

Fig. 23 Feynman diagrams that describe the typical nonrephasing and rephasing pathways in THz-THz-Raman experiments.
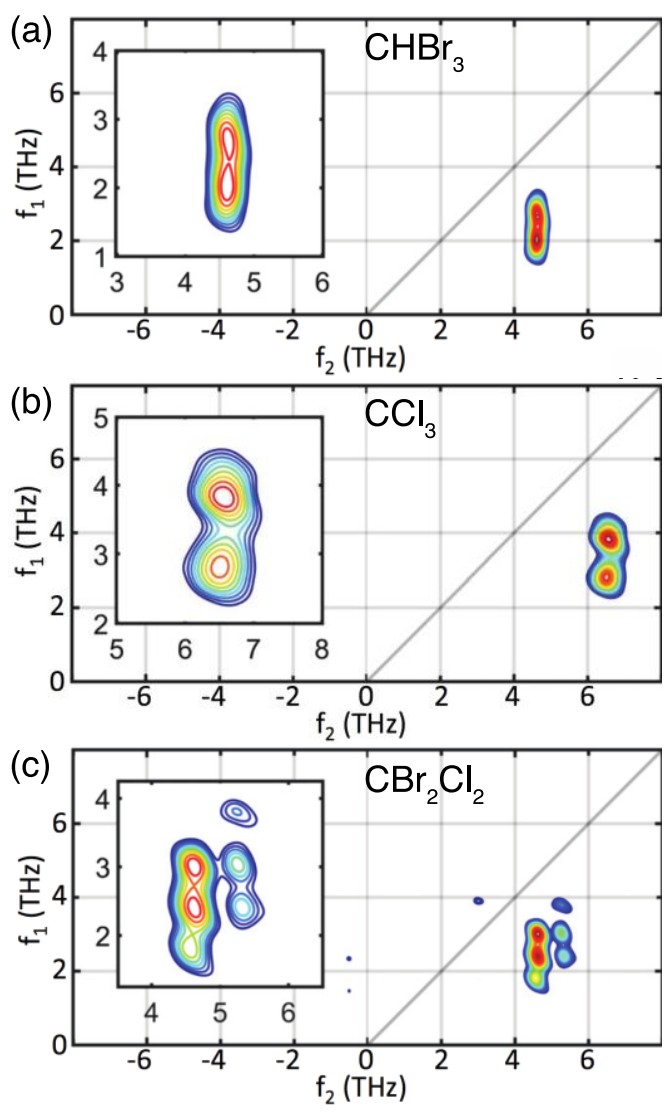

Fig. 24 2D spectra of several simple liquids showing off-diagonal NR peaks originating from anharmonic couplings among various intramolecular vibrations. (from [37]) 
2D THz spectra of several simple liquids are shown in Fig. 24. Off-diagonal peaks from different NR pathways are observed, which clearly show the anharmonic vibrational couplings between different modes in these simple liquids. The experimental data agree well with simulations using the full set of response functions including couplings between different states and all possible pathways as outlined above. The 2D spectra can guide theoretical and computational efforts to elaborate the rich insights into the microscopic dynamics of thermally populated liquid-state vibrational dynamics.

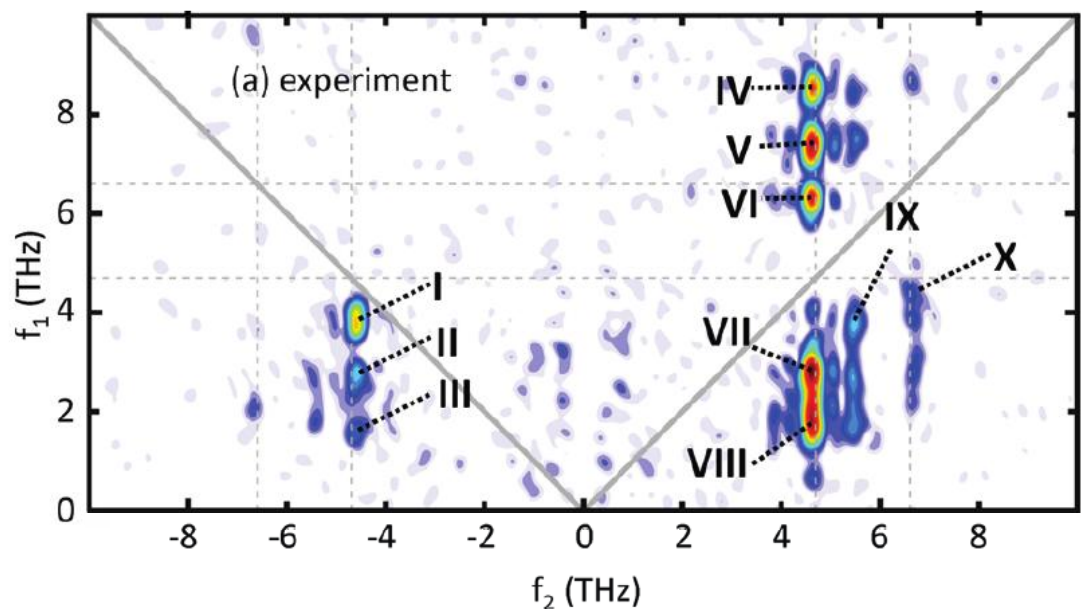

Fig. 25 Experimental 2D THz spectrum from $\mathrm{CHBr}_{3}$. The spectral peaks are labeled. The peaks in the first quadrant are NR signals while those in the second quadrant are $\mathrm{R}$ signals.

Diagonal peaks that involve vibrational population pathways were not observed in this experiment due to the orthogonal polarizations of the input THz pulse pair and the limited $\mathrm{THz}$ bandwidth. Moreover, rephasing signals were not observed due to the limited $\mathrm{THz}$ bandwidth. As shown by the example R pathway in diagram (iii) of Fig. 23, it requires $\mathrm{THz}$ excitation of the transition from $|0\rangle$ to $|2\rangle$, the frequency of which is beyond the available $\mathrm{THz}$ bandwidth in Ref. [37]. In a follow-up work with $\mathrm{THz}$ bandwidth, $\mathrm{R}$ signals were observed [38]. The experimental 2D spectrum of $\mathrm{CHBr}_{3}$ is shown in Fig. 25. In comparison with the spectrum in Fig. 24(a), in addition to the two NR peaks, several NR peaks are observed at higher frequencies in the first quadrant while several $\mathrm{R}$ peaks are observed in the second quadrant. The 2D spectrum reveals the rich anharmonic couplings among different vibrational modes in this simple liquid. The relevant lightmatter interactions can be described by the Feynman pathways shown in Fig. 23.

\subsection{D Raman-THz spectroscopy of water and aqueous salt solutions}

The hydrogen-bond dynamics in water have been explored extensively by 2D IR spectroscopy which focuses on the intramolecular vibrations, for example the $\mathrm{OH}$ stretch mode $[73,74]$. These observations are rather indirect, as information is inferred from the 
IR-frequency spectator modes which are sensitive probes of the strength of hydrogen bonding to the environment, but may not necessarily provide a full picture of the complex collective intermolecular motions of the hydrogen-bond network. The possibility to use 2D Raman- $\mathrm{THz}$ spectroscopy to investigate the hydrogen bond in water has been examined theoretically recently $[75,76]$ as an alternative. THz-frequency intermolecular dynamics of the hydrogen bond, including the librational motion $(\sim 20 \mathrm{THz})$, and the stretch and bend vibrations $(<10 \mathrm{THz})$ [38], may reveal direct information about the molecular dynamics related to the hydrogen bond of water under ambient conditions. Specifically, a photon echo signal investigating the heterogeneity of the hydrogen-bond network would allow one to infer the time scale of a stable network pattern, and thus provide mechanistic insights into the microscopic structure of the hydrogen bond in neat water and aqueous solutions [39].
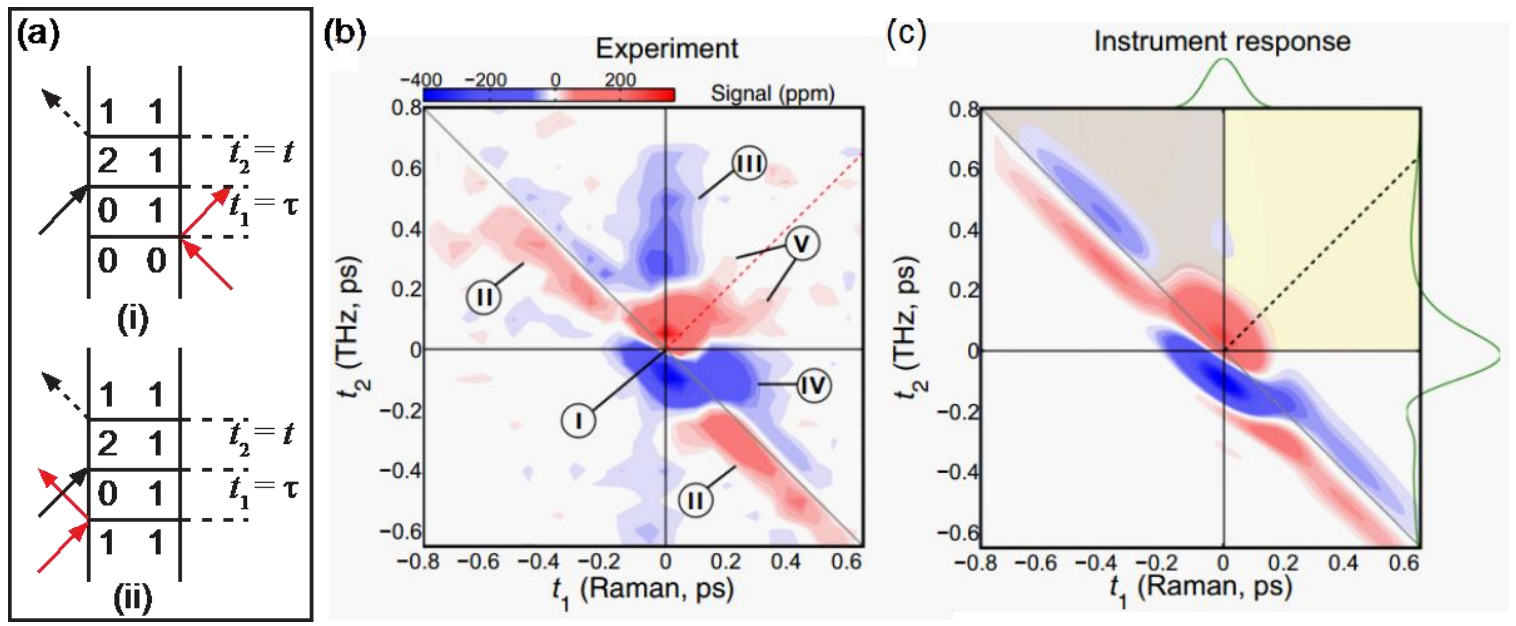

Fig. 26 (a) Feynman diagrams shows the typical rephasing pathways that describes the Raman-THz-THz photon echo signals. The states may denote different states from two intermolecular vibrational modes. (b) 2D Raman- $\mathrm{THz}$ time-domain signal of neat water and (c) the instrument response function. (from [39])

The first 2D Raman-THz spectroscopy experiments have been realized in water and aqueous solutions recently [38], yielding 2D time-domain signals including that shown in Fig. 26. The observation of photon echoes in neat water and various aqueous salt solutions yields the hydrogen-bond relaxation times for these systems. The experimental setup was the same as shown in Fig. 9. The relevant light-matter interaction is elaborated by the pulse sequence shown in Fig. 4(c) and the Feynman diagrams describing typical rephasing pathways shown in Fig. 26(a). For example, in diagram (i), the initial state of the system is a population $|0\rangle\langle 0|$. The Raman excitation pulse induces a first-order vibrational coherence $|0\rangle\langle 1|$ which evolves during time period $\tau$ (or $t_{1}$ in Fig. 26(b)). This $1 \mathrm{QC}$ originates presumably from the broad range of collective intermolecular modes 
involving the hydrogen bond bend and stretch vibrations, and shows rapid decay. The $\mathrm{THz}$ field interacts with the system once, generating a second-order rephasing coherence $|2\rangle\langle 1|$ through a two-quantum transition, for example, to a combination band. The rephasing coherence evolves and emits the nonlinear THz-frequency signals during $t$ (or $t_{2}$ in Fig. 20(b)). The nonlinear signals are detected by EOS. The photon echo signal lies along the diagonal $\tau=t$ (or $t_{1}=t_{2}$ in Fig. 26(b)) in the 2D time-domain trace, i.e. as usual the echo signal emerges after a delay $t$ equal to the inter-pulse delay $\tau$. The $2 \mathrm{D}$ Raman-THz-THz time-domain signal from neat water is shown in Fig. 26(b) in comparison to the instrument response function (IRF) in Fig. 26(c) which assumes the sample response to behave as a $\delta$-function. The difference between them shows evidence of a photon echo signal along the diagonal $\tau=t$ with an average relaxation time of less than 100 fs [39].
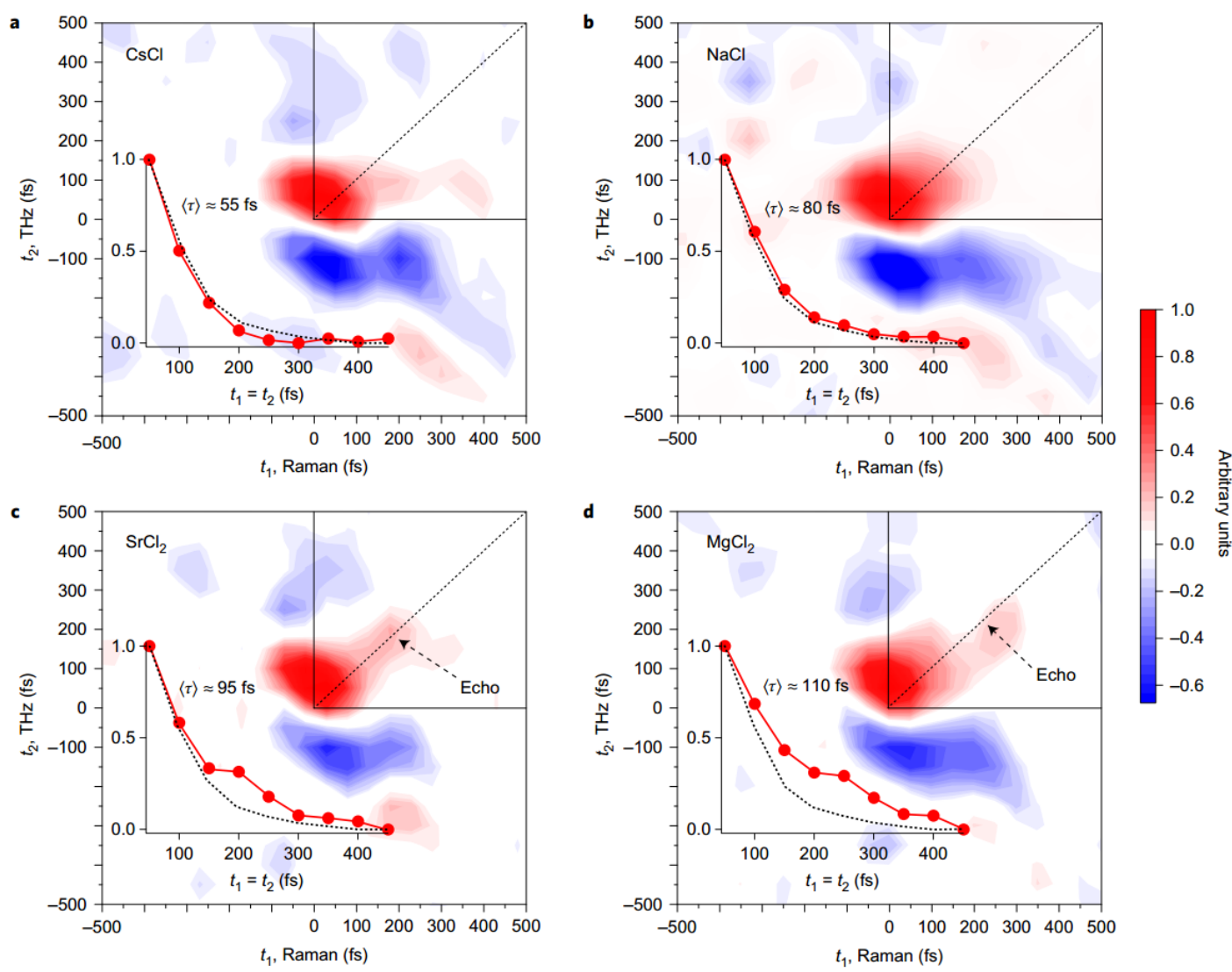

Fig. 27 2D Raman-THz-THz responses of various aqueous salt solutions. The photon echoes lie on the diagonals (dashed lines). The insets show the photon echoes signals as $1 \mathrm{D}$ cuts along the diagonals, which give average relaxation times from different solutions. (from [40])

In a follow-up study [39], several aqueous salt solutions were investigated using the same 2D Raman-THz-THz method. The experimental 2D Raman-THz-THz time-domain 
signals from these solutions are shown in Fig. 27. In these solutions, the presence of different cations changes the viscosity of water presumably by modulating the hydrogenbond network via ion-water interactions. 2D Raman-THz-THz echoes report on the inhomogeneity of the hydrogen-bond network can therefore provide insight into the range of water-ion interactions. The decays of the photon echo signals in these solutions show subtle differences. The average relaxation times extracted from the photon echo signals show a strong correlation with the effects of the corresponding cations on the viscosity of water [40].

\subsection{Extensions of $2 \mathrm{D} \mathrm{THz}$ and $2 \mathrm{D} \mathrm{THz}$-Raman vibrational spectroscopies}

2D spectroscopies utilizing high-frequency $\mathrm{THz}$ pulses and hybrid sequences of $\mathrm{THz}$ and Raman pulses have proved successful in measuring the nonresonant two-phonon coherences in semiconductors, and intramolecular and intermolecular dynamics in liquids including simple halogenated liquids, water, and aqueous salt solutions. The methodology provides new information about low-frequency motions that are important in a wide range of systems including crystalline solids, simple liquids, biomolecules, polymers, glasses, and other materials. All- $\mathrm{THz} 2 \mathrm{D}$ spectroscopy also should prove useful for further progress. Improvements in the excitation $\mathrm{THz}$ field strength and bandwidth [22, 46] as well as data acquisition methods utilizing single-shot signal detection [77] could accelerate the use of these approaches for study of condensed phase molecular dynamics.

\section{2D THz magnetic resonance spectroscopy}

The examples discussed in the above two sections all utilize the THz electric field for 2D spectroscopy of molecular rotational and vibrational degrees of freedom. It is also possible to use the $\mathrm{THz}$ magnetic field to drive the spin degrees of freedom in molecular and condensed matter systems and to conduct 2 $\mathrm{D} \mathrm{THz}$ magnetic resonance spectroscopy, which is directly relevant to chemistry and biology. In this section, we will discuss 2D $\mathrm{THz}$ magnetic resonance spectroscopy and show the first example of its application to collective spin waves (magnons) in a magnetic material.

\subsection{Background and motivation}

Nonlinear manipulation of spins is the basis for all advanced methods in magnetic resonance including multidimensional nuclear magnetic resonance and electron paramagnetic resonance (EPR) spectroscopies [78, 79], magnetic resonance imaging, and, in recent years, quantum control over individual spins [80]. The methodology is 
facilitated by the ease with which the strong-field regime can be reached for radiofrequency or microwave magnetic fields that drive nuclear or electron spins respectively, typified by sequences of magnetic pulses that control the magnetic moment directions [78-80]. The capabilities meet a bottleneck, however, for far-infrared magnetic resonances, which are characteristic of molecular complexes including molecular magnets [81] and metalloproteins [82] containing high-spin transition-metal or rare-earth ions. In these systems, zero-field splittings (ZFSs) due to high magnetic anisotropy and/or spin-spin interactions result in transition frequencies in the $\mathrm{THz}$ frequency region even in the absence of external magnetic fields. Measurements of the spin resonances originating from ZFSs can provide mechanistic insight into molecular magnetic properties and protein catalytic function as the ZFSs show exquisite sensitivity to ligand geometries and transition metal electronic structure. With strong applied magnetic fields $(\sim 10 \mathrm{~T})$, resonances of unpaired electron spins in molecular complexes and metalloproteins can be shifted from the usual microwave regime into the $\mathrm{THz}$ range, thereby drastically improving the resolution of spectral splittings [79, 83, 84].

Despite the critical importance of THz-frequency EPR spectroscopy, current EPR technology remains limited at $\mathrm{THz}$ frequencies because the weak sources used only permit measurements of linear responses, i.e. 1QC or free-induction decay (FID) signals. Utilization of the strong $\mathrm{THz}$ generation techniques discussed in Section 2.1.1 can circumvent this limitation. Nonlinear and 2D THz spectroscopy methods can allow the extension of established, commercially available multidimensional EPR spectroscopy from the microwave to the $\mathrm{THz}$ frequency range. To date, the only available example of 2D THz spectroscopy of the spin degree of freedom was conducted on magnons in a magnetic material [36]. We review it here in the expectation that the methodology will be extended to molecular and biomolecular samples.

\section{$5.22 \mathrm{D} \mathrm{THz}$ spectroscopy of collective spin waves}

Magnons are the elementary excitations in material systems with spin order such as ferromagnetic (FM) and antiferromagnetic (AFM) phases. In these systems, the high magnetic anisotropy and strong spin-spin interactions result in an intrinsic internal magnetic field that is commonly on the order of $10 \mathrm{~T}$. As a result, magnon resonances are usually found in the $\mathrm{THz}$ range. Some of these materials have been studied with continuous-wave and pulsed $\mathrm{THz}$ fields, revealing the magnon frequencies through their FID signals $[85,86]$ and demonstrating linear superposition in the responses to timedelayed pulse pairs $[55,85]$. So far there are very limited examples of nonlinear $\mathrm{THz}$ driving of spins [87-89]. As in other types of 2D spectroscopy, 2D magnetic resonance allows the distinct nonlinear responses to be separated from each other and from linear responses. 
An initial demonstration of $2 \mathrm{D} \mathrm{THz}$ spectroscopy using $\mathrm{THz}$ magnetic fields was conducted on magnons in yttrium ferrite $\left(\mathrm{YFeO}_{3}\right.$ or $\left.\mathrm{YFO}\right)$ which has canted AFM order, as shown in Fig. 22(a). The static spin Hamiltonian describing the two sublattice spins is given by [90, 91],

$$
H_{0}=-J \mathbf{S}_{1} \cdot \mathbf{S}_{2}+\mathbf{D} \cdot\left(\mathbf{S}_{1} \times \mathbf{S}_{2}\right)-\sum_{i=1}^{2}\left(K_{\mathrm{a}} S_{\mathrm{ia}}^{2}+K_{\mathrm{c}} S_{\mathrm{ic}}^{2}\right) .
$$

The first term describes the AFM coupling between neighboring spins $\mathbf{S}_{1}$ and $\mathbf{S}_{2}$ with a positive exchange constant $J$. The second term derives from the Dzyaloshinskii-Moria (DM) spin-spin interaction with the antisymmetric exchange parameter $\mathbf{D}$, a vector along the crystal $b$-axis. As the first term favoring AFM order of the spins is much larger than the second term favoring orthogonal orientation between the two spins, the interplay between them results in the canted AFM order shown in Fig. 28(a) with a canting angle of about $0.45^{\circ}[90,91]$. A net magnetization $\mathbf{M}$ is formed along the crystal $c$ axis due to the canting. The third term accounts for the orthorhombic magnetic anisotropy, which is manifested as the ZFS of the unpaired spins of the high-spin $\mathrm{Fe}^{3+}$, with $K_{\mathrm{a}}$ and $K_{\mathrm{c}}$ the magnetic anisotropy parameters along the crystal $a$ and $c$ axes.

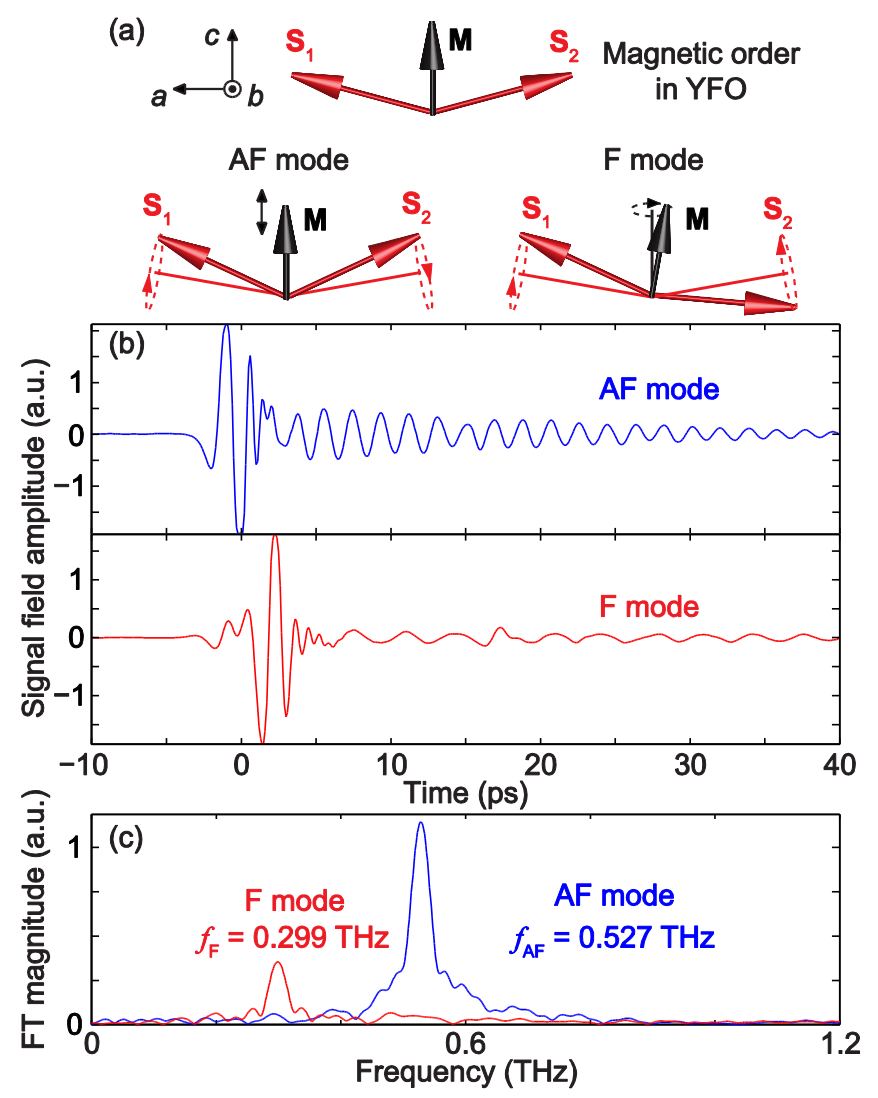

Fig. 28 (a) Spin order and the two magnon modes in YFO. (b) THz fields transmitted through the sample followed by FID signals of each magnon mode. (c) FT magnitude 
spectra of both magnon modes, resulting from a numerical Fourier transformation of the FID signals in (b). (from [36])

The Zeeman interaction between the THz magnetic field $\mathbf{B}_{\mathrm{THz}}$ and the sublattice spins $\mathbf{S}_{1}$ and $\mathbf{S}_{2}$ describes the light-matter interactions. The interaction Hamiltonian $H_{1}$ is given by,

$$
H_{1}=\gamma \mathbf{B}_{\mathrm{THz}} \cdot \sum_{i=1}^{2} \mathbf{S}_{\mathrm{i}},
$$

where $\gamma$ is the gyromagnetic ratio. In the linear response region, two THz-active magnon modes, the quasi-AFM (AF) and quasi-FM (F) modes, can be constructed based respectively on the out-of-phase and in-phase cooperative precessional motions of the sublattice spins. Macroscopically the AF mode corresponds to oscillation of the net magnetization amplitude and the $\mathrm{F}$ mode to precession about the net magnetization direction as shown in Fig. 28(a). In YFO at room temperature, the AF mode at $f_{A F}=$ $0.527 \mathrm{THz}$ and the $\mathrm{F}$ mode at $f_{\mathrm{F}}=0.299 \mathrm{THz}$ can be selectively excited by $\mathrm{THz}$ pulses with magnetic field polarizations parallel and perpendicular respectively to the net magnetization direction. Experimentally this is done by rotating the single-crystal sample while maintaining the polarization directions of the $\mathrm{THz}$ fields. In response to linear $\mathrm{THz}$ excitation, magnons radiate FID signals $B(t)$ at their resonance frequencies, revealing the linear-response spin dynamics. The FID signal fields are measured by EOS and each magnon mode is resolved in the spectrum from Fourier transformation of the FID signals, as shown in Figs. 28(b) and 28(c).

The experimental setup used is identical to that shown in Fig. 6. The sample is an $a$-cut single crystal of YFO. In response to time-delayed strong $\mathrm{THz}$ pulse pair excitation, nonlinear magnon responses are readily revealed in the time-domain signal of $B_{\mathrm{NL}}$. An example of time-domain nonlinear signal measurements of the AF mode is shown in Fig. 29. In this case, the delay $\tau$ is selected to be $3.7 \mathrm{ps}$ which is twice the AF mode period. The magnon responses $B_{A}$ and $B_{B}$ induced by each $\mathrm{THz}$ pulse individually are in phase as shown in Fig. 29(a). As a result, the AF mode response $B_{A B}$ induced by both $\mathrm{THz}$ pulses shows a coherent enhancement in the signal amplitude as shown in Fig. 29(b) [55, 85]. The nonlinear signal $B_{\mathrm{NL}}$ is detected via the differential chopping detection method following $B_{\mathrm{NL}}=B_{A B}-B_{A}-B_{B}$. In the trace of $B_{\mathrm{NL}}$, weak oscillations which have a phase shift of $3 \pi / 2$ relative to $B_{A B}$ and slight asymmetric distortions are observed. The shifted phase is that of a third-order response function and the asymmetry indicates a second-harmonic generation (SHG) signal. Fourier transformation of the nonlinear signal reveals the nonlinear spectrum as shown in Fig. 29(c). A third-order spectral peak is located at the fundamental $\mathrm{AF}$ mode frequency $f_{\mathrm{AF}}$. The peak arises from a sum of the third-order signals resulting from different excitation pathways as detailed below. Magnon SHG signal is located at the second-harmonic AF mode frequency $2 f_{\mathrm{AF}}$. 

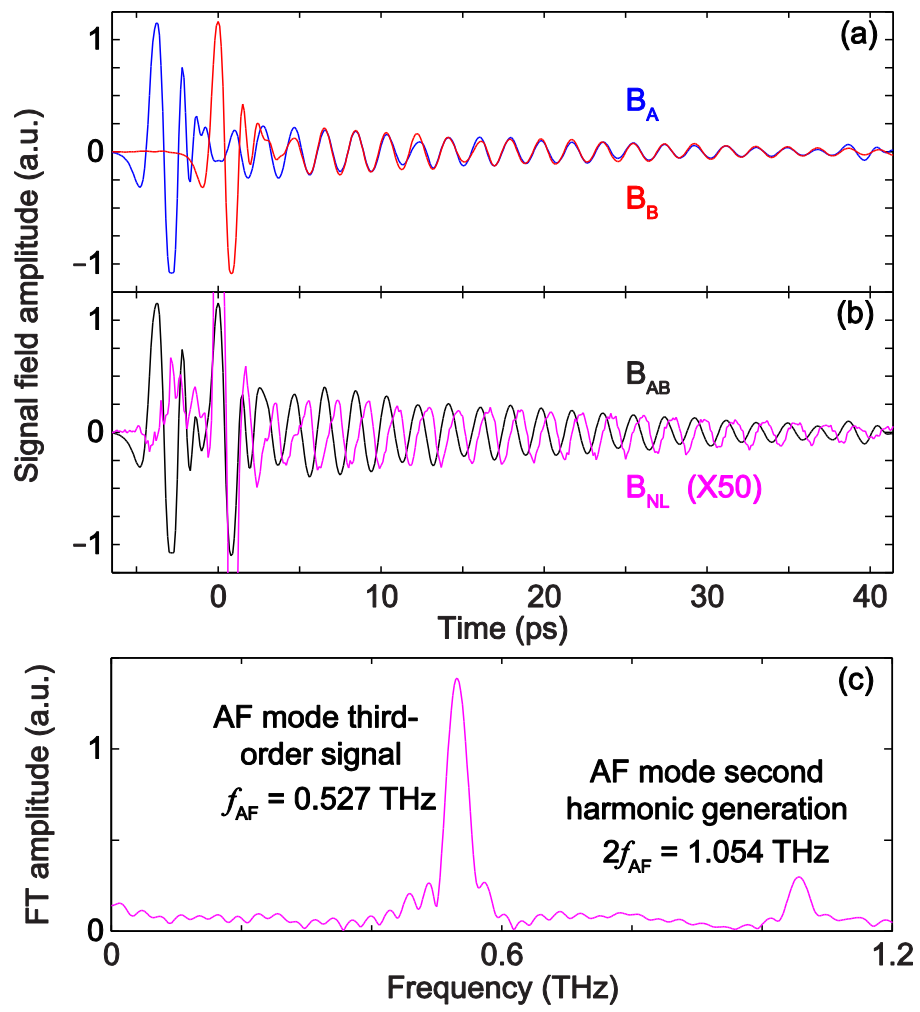

Fig. 29 (a) and (b) Nonlinear signal $B_{\mathrm{NL}}$, extracted by $\mathrm{B}_{\mathrm{NL}}=\mathrm{B}_{A B}-\mathrm{B}_{A}-\mathrm{B}_{B}$, from the AF mode magnons excited with a fixed inter-pulse delay of $\tau=3.7$ ps. (c) Fourier transformation of the oscillatory signals in $B_{\mathrm{NL}}$ reveals the third-order and second-order nonlinear signals at $f_{\mathrm{AF}}$ and $2 f_{\mathrm{AF}}$ respectively. (from [36])

The $2 \mathrm{D} B_{\mathrm{NL}}$ trace of the AF mode was recorded as a function of the inter-pulse delay $\tau$ and detection time $t$ by incrementing $\tau$ with a small step size and recording the $t$ dependent signal $B_{\mathrm{NL}}$ at each $\tau$. Rotating the sample about the crystal $a$ axis allowed excitation of the $\mathrm{F}$ mode, and the $2 \mathrm{D} B_{\mathrm{NL}}$ trace of the $\mathrm{F}$ mode was recorded in the same manner. 2D numerical Fourier transformation of the $B_{\mathrm{NL}}(t, \tau)$ traces with respect to $t$ and $\tau$ generated the 2D complex spectrum of each magnon mode. The 2D magnitude spectra of the two magnon modes in YFO are shown in Fig. 30. As in the 2D rotational spectra, the $2 \mathrm{D}$ magnetic resonance spectra are separated into NR and $\mathrm{R}$ quadrants due to the phase evolution of the magnon coherences during $\tau$ and $t$ in the two different excitation pathways. In each magnon mode, the full set of $\chi^{(3)}$ signals is observed and the R, NR, $2 \mathrm{Q}$ and PP signals appear at easily distinguished locations in the spectra. The relevant THz field-spin interaction pathways are elaborated as follows.

The $\mathrm{R}$ (spin echo) peak and NR peak each result from a single field interaction during pulse A that creates a first-order magnon $1 \mathrm{QC}$ and, after delay $\tau$, two field interactions during pulse $\mathrm{B}$ that generate a magnon population and then a third-order magnon $1 \mathrm{QC}$ (either phase-reversed or not relative to the first-order 1QC) that radiates the nonlinear 
signal. The $2 \mathrm{Q}$ peak arises from two field interactions during pulse A that create a 2QC which accumulates phase at twice the magnon frequency and, after time $\tau$, one field interaction during pulse $\mathrm{B}$ that induces transitions to a third-order 1QC that radiates the signal. The 2Q signal reveals correlations between pairs of zone-center magnons [92] which are distinct from zone-boundary magnon correlations revealed in 2-magnon Raman spectra [93]. The PP signal is generated by two field interactions during pulse A that create magnon population and, after delay $\tau$, one interaction with pulse $\mathrm{B}$ that generates a third-order $1 \mathrm{QC}$ that radiates the signal.

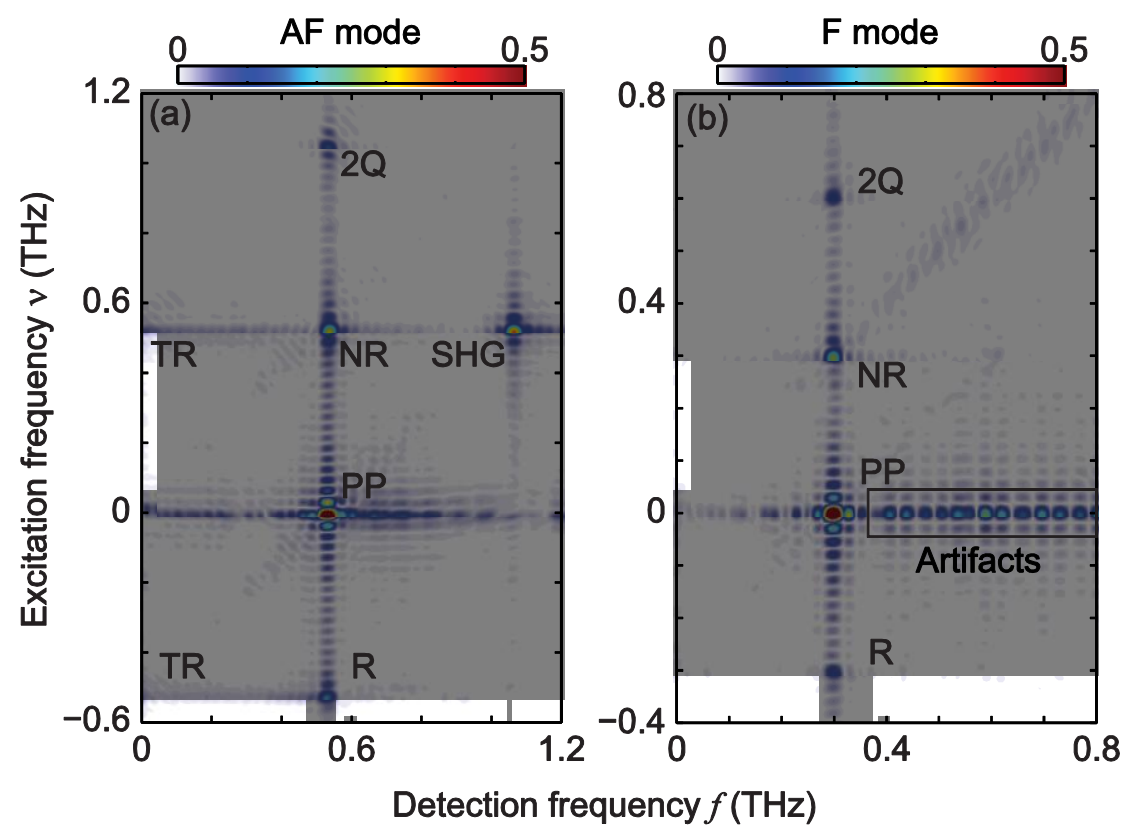

Fig. 30 2D THz magnetic resonance spectra of magnons in YFO. (a) Experimental AF mode 2D magnitude spectrum. Third-order spectral peaks include pump-probe (PP), nonrephasing (NR), rephasing (R), and 2-quantum (2Q) peaks. Second-order peaks include second harmonic generation (SHG) and THz rectification (TR) peaks. (b) Experimental F mode 2D magnitude spectrum showing the full set of third-order peaks. The artifacts are due to signal double-reflections in the sample. Both spectra are normalized and plotted according to the color scale shown. (from [36])

Relevant pathways of the various $\chi^{(3)} \mathrm{THz}$ field-spin interactions described here are further elaborated by the double-sided Feynman diagrams shown in Fig. 31(a). In the 2D spectrum of the AF mode, type-I $\chi^{(2)}$ signals due to $\mathrm{SHG}$ and $\mathrm{THz}$ rectification are also present, which are described by the Feynman diagrams shown in Fig. 31(b). These signals are emitted by a $\chi^{(2)}$ magnetization due to the sum- and difference-frequency mixing of the magnon 1QCs generated by each $\mathrm{THz}$ pulse, which are given by,

$$
M^{(2)}\left(2 \omega_{\mathrm{AF}}\right)=\chi^{(2)}\left(2 \omega_{\mathrm{AF}} ; \omega_{\mathrm{AF}}, \omega_{\mathrm{AF}}\right) B_{A}\left(\omega_{\mathrm{AF}}\right) B_{B}\left(\omega_{\mathrm{AF}}\right) \text {, }
$$




$$
M^{(2)}(0)=\chi^{(2)}\left(0 ;-\omega_{\mathrm{AF}}, \omega_{\mathrm{AF}}\right) B_{A}^{*}\left(\omega_{\mathrm{AF}}\right) B_{B}\left(\omega_{\mathrm{AF}}\right) .
$$

These signals are observed due to the anharmonic precessions of the sublattice spins $\mathbf{S}_{1}$ and $\mathbf{S}_{2}$ whose excursions away from the equilibrium are about $\pm 0.5^{\circ}[88] \cdot \chi^{(2)}$ signals are in principle also observable in the $2 \mathrm{D}$ spectrum of the $\mathrm{F}$ mode. But for the $\mathrm{F}$ mode, the $\chi^{(2)}$ signals that are expected to be parallel to the direction of the net magnetization are perpendicular to the fundamental field polarization. Phase-matching for the type-II $\chi^{(2)}$ process is not satisfied in the thick sample used in this experiment, as the birefringent YFO crystal has a large index difference between the two crystal axes along which the fundamental and $\chi^{(2)}$ magnetic fields are polarized.

\begin{tabular}{|c|c|c|c|c|c|}
\hline (a) $\quad \mathrm{NR}$ & & $\mathrm{R}$ & & (b) SHG & \\
\hline$\because 0$ & & & & 1 & \\
\hline $\begin{array}{ll}10 \\
\end{array}$ & $\gamma_{t_{3}}^{-}=t$ & $\begin{array}{ll}1 & 0 \\
\end{array}$ & $t_{3}=t$ & $\because 00$ & \\
\hline \begin{tabular}{|ll}
1 & 1
\end{tabular} & $\mathrm{t}_{2}=0$ & $\begin{array}{ll}1 & 1\end{array}$ & $\mathrm{t}_{2}=0$ & $\begin{array}{ll}2 & 0 \\
\end{array}$ & $\mathrm{t}_{2}=\mathrm{t}$ \\
\hline $\begin{array}{ll}10 \\
\end{array}$ & & $\begin{array}{ll}0 & 1\end{array}$ & $t_{1}=\tau$ & 10 & $t_{1}=\tau$ \\
\hline $\begin{array}{|ll|}0 & 0\end{array}$ & & $\begin{array}{ll}0 & 0\end{array}$ & & $\begin{array}{ll}0 & 0\end{array}$ & \\
\hline (i) & & (ii) & & (v) & \\
\hline $2 Q$ & & PP & & TR & \\
\hline $0 \quad 0$ & V. & $\begin{array}{ll}0 & 0 \\
\end{array}$ & & $\because \quad 0 \quad 0$ & \\
\hline 10 & $t_{3}=t$ & 100 & $\begin{array}{l}t_{3}=t \\
--\end{array}$ & $\checkmark$ & $\mathrm{t}_{2}=\mathrm{t}$ \\
\hline 20 & $t_{2}=\tau$ & $\begin{array}{ll}1 & 1 \\
\end{array}$ & $\mathrm{t}_{2}=\tau$ & $\begin{array}{ll}1 & 0\end{array}$ & $\mathrm{t}_{1}=\tau$ \\
\hline 10 & $t_{1}=0$ & $\begin{array}{ll}1 & 0 \\
\end{array}$ & $\mathrm{t}_{1}=0$ & $\mid \begin{array}{ll}1 & 0 \\
0 & 0\end{array}$ & \\
\hline $\begin{array}{ll}10 & 0 \\
\text { (iii) }\end{array}$ & & $\begin{array}{ll}0 & 0 \\
\text { (iv) }\end{array}$ & & (vi) & \\
\hline
\end{tabular}

Fig. 31 Double-sided Feynman diagrams show typical excitation pathways leading to the coherent emission of third-order [(a), (i)-(iv)] and second-order [(b) (v)-(vi)] nonlinear signals. (from [36])

\subsection{Extensions of 2D THz EPR spectroscopy}

In this section, we have discussed an example of $2 \mathrm{D} \mathrm{THz}$ magnetic resonance spectroscopy that has directly revealed the full set of $\chi^{(3)}$ nonlinear signals originating from the magnons in a magnetic material. The methodology is expected to be applicable to the study of many chemical and biological systems with spin resonances at $\mathrm{THz}$ frequencies. To date, linear THz-frequency EPR spectroscopy has been conducted based on $\mathrm{THz}$ time-domain spectroscopy [94, 95], coherent synchrotron radiation [96] or blackbody radiation [97, 98]. These studies have measured the ZFSs in single-molecule magnets $[95,96]$ and other molecular and biological systems $[95,99,100]$ at zero and 
nonzero external magnetic field. We anticipate that 2D EPR spectroscopy of molecular complexes and biomolecules in the $\mathrm{THz}$ frequency range will be demonstrated and will provide wide-ranging new insights just as it has in lower frequencies.

\section{Conclusion and outlook}

Utilizing existing $\mathrm{THz}$ spectroscopic techniques, it is now possible to record $2 \mathrm{D} \mathrm{THz}$ spectra originating from various material degrees of freedom and phases, including the

rotations of gas-phase molecules, spin precessions in a magnetic crystal, and intra- and intermolecular dynamics in liquids. We note again that we have not reviewed $2 \mathrm{D} \mathrm{THz}$ or multi-THz spectroscopy of electronic responses [27, 29, 30, 101]. Even including those examples, it is evident that $2 \mathrm{D} \mathrm{THz}$ spectroscopy is still in a very nascent stage. The use of recently developed nonlinear optical crystals [22-24, 102] for generation of strong $\mathrm{THz}$ fields that span a wide frequency range, extending all the way to the longwavelength IR region, will substantially increase the scope of $2 \mathrm{D} \mathrm{THz}$ studies. Further use of frequency-selected and otherwise tailored THz fields [103-105], a third THz pulse $[33,34]$ to establish an additional variable time period, multiple $\mathrm{THz}$ sources or beam paths to permit non-collinear $\mathrm{THz}$ field polarizations [37] and combined $\mathrm{THz}$ and optical fields for signal generation and detection $[37,60]$ will extend the information content that can be extracted in a wide range of samples. Incorporating single-shot $\mathrm{THz}$ detection methods [77] could drastically reduce the long data acquisition times which presently hinder virtually all 2D THz measurements. As in other frequency ranges including radio, microwave, infrared and visible, multidimensional spectroscopy in the $\mathrm{THz}$ regime can reveal otherwise elusive structure, interactions, and dynamics. With further advances in the relevant technologies and refinements of the measurement methodology, $2 \mathrm{D} \mathrm{THz}$ spectroscopy can be expected to take its place among the multidimensional spectroscopies that have produced broad and deep advances in all areas of chemistry.

\section{Acknowledgements}

We thank Sharly Fleischer, Takayuki Kurihara, and Tohru Suemoto for contributions to this work. This work was supported, in part, by Office of Naval Research Grant N0001413-1-0509 and Defense University Research Instrumentation Program Grant N00014-151-2879, National Science Foundation Grant CHE-1111557, and the Samsung Global Research Outreach program. 


\section{References}

1. Hamm P, Zanni MT (2011) Concepts and methods of 2D infrared spectroscopy. Cambridge University Press

2. Mukamel S (2000) Multidimensional femtosecond correlation spectroscopies of electronic and vibrational excitations. Annu Rev Phys Chem 51:691-729 . doi: 10.1146/annurev.physchem.51.1.691

3. Jonas DM (2003) Two-dimensional femtosecond spectroscopy. Annu Rev Phys Chem 54:425-463 . doi: 10.1146/annurev.physchem.54.011002.103907

4. Zheng J, Kwak K, Fayer MD (2007) Ultrafast 2D IR vibrational echo spectroscopy. Acc Chem Res 40:75-83 . doi: 10.1021/ar068010d

5. Ramasesha K, De Marco L, Mandal A, Tokmakoff A (2013) Water vibrations have strongly mixed intra- and intermolecular character. Nat Chem 5:935-940 . doi: $10.1038 /$ nchem. 1757

6. Baiz CR, Reppert M, Tokmakoff A (2013) Amide I Two-Dimensional Infrared Spectroscopy: Methods for Visualizing the Vibrational Structure of Large Proteins. J Phys Chem A 117:5955-5961 . doi: 10.1021/jp310689a

7. Krummel AT, Mukherjee P, Zanni MT (2003) Inter and Intrastrand Vibrational Coupling in DNA Studied with Heterodyned 2D-IR Spectroscopy. J Phys Chem B 107:9165-9169 . doi: 10.1021/JP035473H

8. Fuller FD, Ogilvie JP (2015) Experimental implementations of two-dimensional Fourier transform electronic spectroscopy. Annu Rev Phys Chem 66:667-690 . doi: 10.1146/annurev-physchem-040513-103623

9. Stone KW, Gundogdu K, Turner DB, et al (2009) Two-Quantum 2D FT Electronic Spectroscopy of Biexcitons in GaAs Quantum Wells. Science (80- ) 324:11691173 . doi: $10.1126 /$ science. 1170274

10. Turner DB, Nelson KA (2010) Coherent measurements of high-order electronic correlations in quantum wells. Nature 466:1089-1092 . doi: 10.1038/nature09286

11. Brixner T, Stenger J, Vaswani HM, et al (2005) Two-dimensional spectroscopy of electronic couplings in photosynthesis. Nature 434:625-628 . doi: 10.1038/nature03429

12. Cheng Y-C, Fleming GR (2009) Dynamics of light harvesting in photosynthesis. Annu Rev Phys Chem 60:241-262 . doi: 10.1146/annurev.physchem.040808.090259

13. Eisele DM, Arias DH, Fu X, et al (2014) Robust excitons inhabit soft supramolecular nanotubes. Proc Natl Acad Sci 111:E3367-E3375 . doi: 10.1073/pnas. 1408342111

14. Krebs N, Pugliesi I, Hauer J, Riedle E (2013) Two-dimensional Fourier transform spectroscopy in the ultraviolet with sub-20 fs pump pulses and 250-720 nm 
supercontinuum probe. New J Phys 15:85016 . doi: 10.1088/13672630/15/8/085016

15. Lee Y-S (2009) Principles of terahertz science and technology. Springer

16. Blanchard F, Razzari L, Bandulet HC, et al (2007) Generation of $1.5 \mu \mathrm{J}$ singlecycle terahertz pulses by optical rectification from a large aperture ZnTe crystal. Opt Express 15:13212 . doi: 10.1364/OE.15.013212

17. Ferguson B, Zhang X-C (2002) Materials for terahertz science and technology. Nat Mater 1:26-33 . doi: 10.1038/nmat708

18. Schmuttenmaer CA (2004) Exploring dynamics in the far-infrared with terahertz spectroscopy. Chem Rev 104:1759-1779 . doi: 10.1021/cr020685g

19. Tonouchi M (2007) Cutting-edge terahertz technology. Nat Photonics 1:97-105 . doi: 10.1038/nphoton.2007.3

20. Yeh KL, Hoffmann MC, Hebling J, Nelson KA (2007) Generation of $10 \mu \mathrm{J}$ ultrashort terahertz pulses by optical rectification. Appl Phys Lett 90:171121 . doi: $10.1063 / 1.2734374$

21. Hirori H, Doi A, Blanchard F, Tanaka K (2011) Single-cycle terahertz pulses with amplitudes exceeding $1 \mathrm{MV} / \mathrm{cm}$ generated by optical rectification in LiNbO3. Appl Phys Lett 98:91106 . doi: 10.1063/1.3560062

22. Shalaby M, Hauri CP (2015) Demonstration of a low-frequency three-dimensional terahertz bullet with extreme brightness. Nat Commun 6:5976 . doi: 10.1038/ncomms6976

23. Vicario C, Ruchert C, Hauri CP (2015) High field broadband $\mathrm{THz}$ generation in organic materials. J Mod Opt 62:1480-1485 . doi: 10.1080/09500340.2013.800242

24. Lee S-H, Jazbinsek M, Hauri CP, Kwon O-P (2016) Recent progress in acentric core structures for highly efficient nonlinear optical crystals and their supramolecular interactions and terahertz applications. CrystEngComm 18:71807203 . doi: 10.1039/C6CE00707D

25. Kampfrath T, Tanaka K, Nelson KA (2013) Resonant and nonresonant control over matter and light by intense terahertz transients. Nat Photonics 7:680-690 . doi: 10.1038/nphoton.2013.184

26. Hwang HY, Fleischer S, Brandt NC, et al (2015) A review of non-linear terahertz spectroscopy with ultrashort tabletop-laser pulses. J Mod Opt 62:1447-1479 . doi: 10.1080/09500340.2014.918200

27. Woerner M, Kuehn W, Bowlan P, et al (2013) Ultrafast two-dimensional terahertz spectroscopy of elementary excitations in solids. New J Phys 15:25039 . doi: $10.1088 / 1367-2630 / 15 / 2 / 025039$

28. Kuehn W, Reimann K, Woerner M, et al (2011) Strong correlation of electronic and lattice excitations in $\mathrm{GaAs} / \mathrm{AlGaAs}$ semiconductor quantum wells revealed by two-dimensional terahertz spectroscopy. Phys Rev Lett 107:67401 . doi: 10.1103/PhysRevLett.107.067401 
29. Somma C, Reimann K, Flytzanis C, et al (2014) High-field terahertz bulk photovoltaic effect in lithium niobate. Phys Rev Lett 112:146602 . doi: 10.1103/PhysRevLett.112.146602

30. Maag T, Bayer A, Baierl S, et al (2015) Coherent cyclotron motion beyond Kohn's theorem. Nat Phys 11:1-6 . doi: 10.1038/NPHYS3559

31. Lu J, Zhang Y, Hwang HY, et al (2016) Nonlinear two-dimensional terahertz photon echo and rotational spectroscopy in the gas phase. Proc Natl Acad Sci USA 113:11800-11805 . doi: 10.1073/pnas.1609558113

32. Lu J, Zhang Y, Hwang HY, et al (2016) Two-dimensional Terahertz Photon Echo and Rotational Spectroscopy in the Gas Phase. In: International Conference on Ultrafast Phenomena. OSA, Washington, D.C., p UTu1A.6

33. Somma C, Folpini G, Reimann K, et al (2016) Two-Phonon Quantum Coherences in Indium Antimonide Studied by Nonlinear Two-Dimensional Terahertz Spectroscopy. Phys Rev Lett 116:1-6 . doi: 10.1103/PhysRevLett.116.177401

34. Somma C, Folpini G, Reimann K, et al (2016) Phase-resolved two-dimensional terahertz spectroscopy including off-resonant interactions beyond the $\chi^{(3)}$ limit. $\mathbf{J}$ Chem Phys 144:184202 . doi: 10.1063/1.4948639

35. Lu J, Li X, Hwang HY, et al (2016) 2D Nonlinear Terahertz Magnetic Resonance Spectroscopy of Magnons in a Canted Antiferromagnet. Int Conf Ultrafast Phenom 9-10 . doi: 10.1364/UP.2016.UTh3A.2

36. Lu J, Li X, Hwang HY, et al (2017) Coherent two-dimensional terahertz magnetic resonance spectroscopy of collective spin waves. Phys Rev Lett 118:207204 . doi: 10.1103/PhysRevLett.118.207204

37. Finneran IA, Welsch R, Allodi MA, et al (2016) Coherent two-dimensional terahertz-terahertz-Raman spectroscopy. Proc Natl Acad Sci USA 113:6857-6861 . doi: 10.1073/pnas.1605631113

38. Finneran IA, Welsch R, Allodi MA, et al (2017) 2D THz-THz-Raman PhotonEcho Spectroscopy of Molecular Vibrations in Liquid Bromoform. J Phys Chem Lett 8:4640-4644 . doi: 10.1021/acs.jpclett.7b02106

39. Savolainen J, Ahmed S, Hamm P (2013) Two-dimensional Raman-terahertz spectroscopy of water. Proc Natl Acad Sci U S A 110:20402-20407 . doi: 10.1073/pnas. 1317459110

40. Shalit A, Ahmed S, Savolainen J, Hamm P (2016) Terahertz echoes reveal the inhomogeneity of aqueous salt solutions. Nat Chem 9:273-278 . doi: $10.1038 /$ nchem. 2642

41. Boyd R (2007) Nonlinear optics. Academic Press

42. Hebling J, Almasi G, Kozma I, Kuhl J (2002) Velocity matching by pulse front tilting for large area THz-pulse generation. Opt Express 10:1161 . doi: 10.1364/OE.10.001161

43. Hebling J, Yeh K-L, Hoffmann MC, et al (2008) Generation of high-power 
terahertz pulses by tilted-pulse-front excitation and their application possibilities. J Opt Soc Am B 25:B6-B19 . doi: 10.1364/JOSAB.25.0000B6

44. Vicario C, Jazbinsek M, Ovchinnikov A V., et al (2015) High efficiency $\mathrm{THz}$ generation in DSTMS, DAST and OH1 pumped by Cr:forsterite laser. Opt Express 23:4573-4580 . doi: 10.1364/OE.23.004573

45. Dai J, Liu J, Zhang XC (2011) Terahertz wave air photonics: Terahertz wave generation and detection with laser-induced gas plasma. IEEE J Sel Top Quantum Electron 17:183-190 . doi: 10.1109/JSTQE.2010.2047007

46. Clough B, Dai J, Zhang XC (2012) Laser air photonics: Beyond the terahertz gap. Mater. Today 15:50-58

47. Carr GL, Martin MC, McKinney WR, et al (2002) High-power terahertz radiation from relativistic electrons. Nature 420:153-6 . doi: 10.1038/nature01175

48. Wu Z, Fisher AS, Goodfellow J, et al (2013) Intense terahertz pulses from SLAC electron beams using coherent transition radiation. Rev Sci Instrum 84:22701 . doi: 10.1063/1.4790427

49. Wu Q, Zhang XC (1995) Free-space electro-optic sampling of terahertz beams. Appl Phys Lett 67:3523 . doi: 10.1063/1.114909

50. Nahata A, Auston DH, Heinz TF, Wu C (1996) Coherent detection of freely propagating terahertz radiation by electro-optic sampling. Appl Phys Lett 68:150 . doi: $10.1063 / 1.116130$

51. Novelli F, Fausti D, Giusti F, et al (2013) Mixed regime of light-matter interaction revealed by phase sensitive measurements of the dynamical Franz-Keldysh effect. Sci Rep 3:1227 . doi: 10.1038/srep01227

52. Pein BC, Chang W, Hwang HY, et al (2016) Terahertz-driven Luminescence and Colossal Stark Effect in CdSe:CdS Colloidal Quantum Dots. Submitted

53. Cook DJ, Chen JX, Morlino EA, Hochstrasser RM (1999) Terahertz-field-induced second-harmonic generation measurements of liquid dynamics. Chem Phys Lett 309:221-228 . doi: 10.1016/S0009-2614(99)00668-5

54. Hoffmann MC, Brandt NC, Hwang HY, et al (2009) Terahertz Kerr effect. Appl Phys Lett 95:231105 . doi: 10.1063/1.3271520

55. Kampfrath T, Sell A, Klatt G, et al (2011) Coherent terahertz control of antiferromagnetic spin waves. Nat Photonics 5:31-34 . doi: 10.1038/nphoton.2010.259

56. Allodi MA, Finneran IA, Blake GA (2015) Nonlinear terahertz coherent excitation of vibrational modes of liquids. J Chem Phys 143:234204 . doi: $10.1063 / 1.4938165$

57. Hwang HY, Hoffmann MC, Brandt NC, Nelson KA (2010) THz Kerr Effect in Relaxor Ferroelectrics. Phys Rev Lett 7-8 . doi: 10.1364/UP.2010.ThE41

58. Lu J, Li X, Hwang HY, et al (2016) Terahertz Kerr Effect in an Organic Ferroelectric. Int Conf Ultrafast Phenom 9-10 . doi: 10.1364/UP.2016.UW4A.14 
59. Fleischer S, Zhou Y, Field RW, Nelson KA (2011) Molecular orientation and alignment by intense single-cycle THz pulses. Phys Rev Lett 107:163603 . doi: 10.1103/PhysRevLett.107.163603

60. Fleischer S, Field RW, Nelson KA (2012) Commensurate two-quantum coherences induced by time-delayed THz fields. Phys Rev Lett 109:123603 . doi: 10.1103/PhysRevLett.109.123603

61. Hamm P (2005) Principles of Nonlinear Optical Spectroscopy: A Practical Approach or: Mukamel for Dummies. In: Univ. Zurich. http://www.mitr.p.lodz.pl/evu/lectures/Hamm.pdf

62. Tokmakoff A (2014) Time-Dependent Quantum Mechanics and Spectroscopy. In: Univ. Chicago. http://tdqms.uchicago.edu/

63. Hamm P, Shalit A (2017) Perspective: Echoes in 2D-Raman-THz spectroscopy. J Chem Phys 146:130901 . doi: 10.1063/1.4979288

64. Tokmakoff A, Lang M, Larsen D, et al (1997) Two-Dimensional Raman Spectroscopy of Vibrational Interactions in Liquids. Phys Rev Lett 79:2702-2705 . doi: 10.1103/PhysRevLett.79.2702

65. Frostig H, Bayer T, Dudovich N, et al (2015) Single-beam spectrally controlled two-dimensional Raman spectroscopy. Nat Photonics 9:339-343 . doi: 10.1038/nphoton.2015.64

66. Harde H, Keiding S, Grischkowsky D (1991) THz commensurate echoes: Periodic rephasing of molecular transitions in free-induction decay. Phys Rev Lett 66:1834-1837 . doi: 10.1103/PhysRevLett.66.1834

67. Fleischer S, Averbukh IS, Prior Y (2007) Selective alignment of molecular spin isomers. Phys Rev Lett 99:93002 . doi: 10.1103/PhysRevLett.99.093002

68. Karras G, Hertz E, Billard F, et al (2015) Orientation and alignment echoes. Phys Rev Lett 114:153601 . doi: 10.1103/PhysRevLett.114.153601

69. Engel GS, Calhoun TR, Read EL, et al (2007) Evidence for wavelike energy transfer through quantum coherence in photosynthetic systems. Nature 446:782786 . doi: $10.1038 /$ nature 05678

70. Roberts ST, Loparo JJ, Tokmakoff A (2006) Characterization of spectral diffusion from two-dimensional line shapes. J Chem Phys 125:84502 . doi: $10.1063 / 1.2232271$

71. Damari R, Kallush S, Fleischer S (2016) Rotational Control of Asymmetric Molecules: Dipole- versus Polarizability-Driven Rotational Dynamics. Phys Rev Lett 117:103001 . doi: 10.1103/PhysRevLett.117.103001

72. Damari R, Rosenberg D, Fleischer S (2017) Coherent Radiative Decay of Molecular Rotations: A Comparative Study of Terahertz-Oriented versus Optically Aligned Molecular Ensembles. Phys Rev Lett 119:33002 . doi: 10.1103/PhysRevLett.119.033002

73. Fecko, C. J., Eaves, J. D., Loparo, J. J., Tokmakoff, A., Geissler PL (2003) 
Ultrafast Hydrogen-Bond Dynamics in the Infrared Spectroscopy of Water. Science (80- ) 301:1698-1702 . doi: 10.1126/science.1087251

74. Asbury JB, Steinel T, Kwak K, et al (2004) Dynamics of water probed with vibrational echo correlation spectroscopy. J Chem Phys 121:12431-12446 . doi: 10.1063/1.1818107

75. Hamm P, Savolainen J (2012) Two-dimensional-Raman-terahertz spectroscopy of water: Theory. J Chem Phys 136:94516 . doi: 10.1063/1.3691601

76. Hamm P, Savolainen J, Ono J, Tanimura Y (2012) Note: Inverted time-ordering in two-dimensional-Raman-terahertz spectroscopy of water. J. Chem. Phys. 136:236101

77. Teo SM, Ofori-Okai BK, Werley CA, Nelson KA (2015) Invited Article: Singleshot $\mathrm{THz}$ detection techniques optimized for multidimensional $\mathrm{THz}$ spectroscopy. Rev Sci Instrum 86:51301 . doi: 10.1063/1.4921389

78. Bajaj VS, Mak-Jurkauskas ML, Belenky M, et al (2009) Functional and shunt states of bacteriorhodopsin resolved by $250 \mathrm{GHz}$ dynamic nuclear polarizationenhanced solid-state NMR. Proc Natl Acad Sci U S A 106:9244-9249 . doi: 10.1073/pnas.0900908106

79. Borbat PP, Costa-Filho AJ, Earle KA, et al (2001) Electron spin resonance in studies of membranes and proteins. Science (80- ) 291:266-269 . doi: 10.1126/science.291.5502.266

80. Koppens FHL, Buizert C, Tielrooij KJ, et al (2006) Driven coherent oscillations of a single electron spin in a quantum dot. Supplementary Notes. Nature 442:766-71 . doi: 10.1038/nature05065

81. Craig GA, Murrie M (2015) 3d single-ion magnets. Chem Soc Rev 44:2135-2147 . doi: $10.1039 / \mathrm{C} 4 \mathrm{CS} 00439 \mathrm{~F}$

82. Nehrkorn J, Martins BM, Holldack K, et al (2013) Zero-field splittings in metHb and metMb with aquo and fluoro ligands: a FD-FT THz-EPR study. Mol Phys 111:2696-2707 . doi: 10.1080/00268976.2013.809806

83. Andersson KK, Schmidt PP, Katterle B, et al (2003) Examples of high-frequency EPR studies in bioinorganic chemistry. J Biol Inorg Chem 8:235-247 . doi: 10.1007/s00775-002-0429-0

84. Möbius K, Savitsky A, Wegener C, et al (2005) Combining high-field EPR with site-directed spin labeling reveals unique information on proteins in action. Magn Reson Chem 43:S4-S19 . doi: 10.1002/mrc.1690

85. Yamaguchi K, Nakajima M, Suemoto T (2010) Coherent control of spin precession motion with impulsive magnetic fields of half-cycle terahertz radiation. Phys Rev Lett 105:237201 . doi: 10.1103/PhysRevLett.105.237201

86. Kozlov G V., Lebedev SP, Mukhin AA, et al (1993) Submillimeter backwardwave oscillator spectroscopy of the rare-earth orthoferrites. IEEE Trans Magn 29:3443-3445 . doi: 10.1109/20.281190 
87. Baierl S, Hohenleutner M, Kampfrath T, et al (2016) Nonlinear spin control by terahertz-driven anisotropy fields. Nat Photonics 10:715-718 . doi: 10.1038/nphoton.2016.181

88. Baierl S, Mentink JH, Hohenleutner M, et al (2016) Terahertz-driven nonlinear spin response of antiferromagnetic nickel oxide. Phys Rev Lett 117:197201 . doi: 10.1103/PhysRevLett.117.197201

89. Mukai Y, Hirori H, Yamamoto T, et al (2016) Nonlinear magnetization dynamics of antiferromagnetic spin resonance induced by intense terahertz magnetic field. New J Phys 18:13045 . doi: 10.1088/1367-2630/18/1/013045

90. Herrmann GF (1963) Resonance and high frequency susceptibility in canted antiferromagnetic substances. J Phys Chem Solids 24:597-606 . doi: $10.1016 / \mathrm{S} 0022-3697(63) 80001-3$

91. Herrmann GF (1964) Magnetic resonances and susceptibility in orthoferrites. Phys Rev 133:A1334-A1344 . doi: 10.1103/PhysRev.133.A1334

92. Morello A, Stamp PCE, Tupitsyn IS (2006) Pairwise decoherence in coupled spin qubit networks. Phys Rev Lett 97:207206 . doi: 10.1103/PhysRevLett.97.207206

93. Fleury PA, Loudon R (1968) Scattering of light by one- and two-magnon excitations. Phys Rev 166:514-530 . doi: 10.1103/PhysRev.166.514

94. Kozuki K, Nagashima T, Hangyo M (2011) Measurement of electron paramagnetic resonance using terahertz time-domain spectroscopy. Opt Express 19:24950 . doi: 10.1364/OE.19.024950

95. Lu J, Li X, Skorupskii G, et al (2017) Rapid and Precise Determination of Absolute Zero-Field Splittings by Terahertz Time-Domain Electron Paramagnetic Resonance Spectroscopy. Chem Sci 1-21 . doi: 10.1039/C7SC00830A

96. Schnegg A, Behrends J, Lips K, et al (2009) Frequency domain Fourier transform THz-EPR on single molecule magnets using coherent synchrotron radiation. Phys Chem Chem Phys 11:6820-25 . doi: 10.1039/b905709a

97. Champion PM, Sievers AJ (1977) Far infrared magnetic resonance in $\mathrm{FeSiF}_{6} \cdot 6 \mathrm{H}$ ${ }_{2} \mathrm{O}$ and $\mathrm{Fe}(\mathrm{SPh}){ }_{4}{ }^{2-}$. J Chem Phys 66:1819-1825 . doi: 10.1063/1.434200

98. Brackett GC (1971) Far-Infrared Magnetic Resonance in Fe(III) and Mn(III) Porphyrins, Myoglobin, Hemoglobin, Ferrichrome A, and $\mathrm{Fe}(\mathrm{III})$ Dithiocarbamates. J Chem Phys 54:4383 . doi: 10.1063/1.1674688

99. Nehrkorn J, Martins BM, Holldack K, et al (2013) Zero-field splittings in metHb and metMb with aquo and fluoro ligands: a FD-FT THz-EPR study. Mol Phys 111:2696-2707 . doi: 10.1080/00268976.2013.809806

100. Nehrkorn J, Telser J, Holldack K, et al (2015) Simulating Frequency-Domain Electron Paramagnetic Resonance: Bridging the Gap between Experiment and Magnetic Parameters for High-Spin Transition-Metal Ion Complexes. J Phys Chem B 119:13816-13824 . doi: 10.1021/acs.jpcb.5b04156

101. Kuehn W, Reimann K, Woerner M, et al (2011) Two-dimensional terahertz 
correlation spectra of electronic excitations in semiconductor quantum wells. $\mathbf{J}$ Phys Chem B 115:5448-5455 . doi: 10.1021/jp1099046

102. Lee S-H, Lu J, Lee S-J, et al (2017) Benzothiazolium Single Crystals: A New Class of Nonlinear Optical Crystals with Efficient $\mathrm{THz}$ Wave Generation. Adv Mater 29:1701748 . doi: 10.1002/adma.201701748

103. Chen Z, Zhou X, Werley CA, Nelson KA (2011) Generation of high power tunable multicycle teraherz pulses. Appl Phys Lett 99:71102 . doi: 10.1063/1.3624919

104. Lu J, Hwang HY, Li X, et al (2015) Tunable multi-cycle THz generation in organic crystal HMQ-TMS. Opt Express 23:22723-22729 . doi: 10.1364/OE.23.022723

105. Liu B, Bromberger H, Cartella A, et al (2017) Generation of narrowband, highintensity, carrier-envelope phase-stable pulses tunable between 4 and $18 \mathrm{THz}$. Opt Lett 42:129 . doi: 10.1364/OL.42.000129 